\title{
Influence of turbulence closure models on the vortical flow field around a submarine body undergoing steady drift
}

\author{
Alexander B. Phillips · Stephen R. Turnock - Maaten Furlong
}

the date of receipt and acceptance should be inserted later

\begin{abstract}
Manoeuvring underwater vehicles experience complex three dimensional flow. Features include stagnation and boundary layer separation along a convex surface. The resulting free vortex sheet rolls up to form a pair of streamwise body vortices. The track and strength of the body vortex pair results in a nonlinear increase in lift with as body incidence increases. Consequently, accurate capture of the body vortex pair is essential if the flow field around a manoeuvring submarine and the resulting hydrodynamic loading is to be correctly found. This work highlights the importance of both grid convergence and turbulence closure models on the strength and path of the crossflow induced body vortices experienced by the DOR submarine model at an incidence angle of $15^{\circ}$. Five turbulence closure models are considered; Spalart-Allmaras, $k-\varepsilon, k-\omega$, Shear Stress Transport and the SSG Reynolds stress model. The SSG Reynolds stress model shows potential improvements in predicting both the path and strength of the body vortex over standard one and two equation turbulence closure models based on an eddy viscosity approach.
\end{abstract}

Keywords CFD · Manoeuvring · Submarine .

Vortex Structure · Turbulence Closure Model

\section{Introduction}

Pitched axi-symmetric bodies, such as an underwater vehicle in a depth change manoeuvre, experience complex three dimensional flows. Four distinct flow regimes at incidence angles of $0^{\circ}$ to $90^{\circ}$ are described in [1], reflecting the decreasing axial flow component. Firstly, at small angles the flow remains attached and the axial flow regime dominates, lift forces are linearly

\footnotetext{
A. B. Phillips

University of Southampton, UK

Tel.: +44-2380-596616

Fax: +44-2380-593299

E-mail: abp@soton.ac.uk

S.R. Turnock

University of Southampton, UK

M. Furlong
}

National Oceanography Centre, Southampton, UK related to incidence angle, thus hull loading should be well predicted using linear hydrodynamic derivatives.

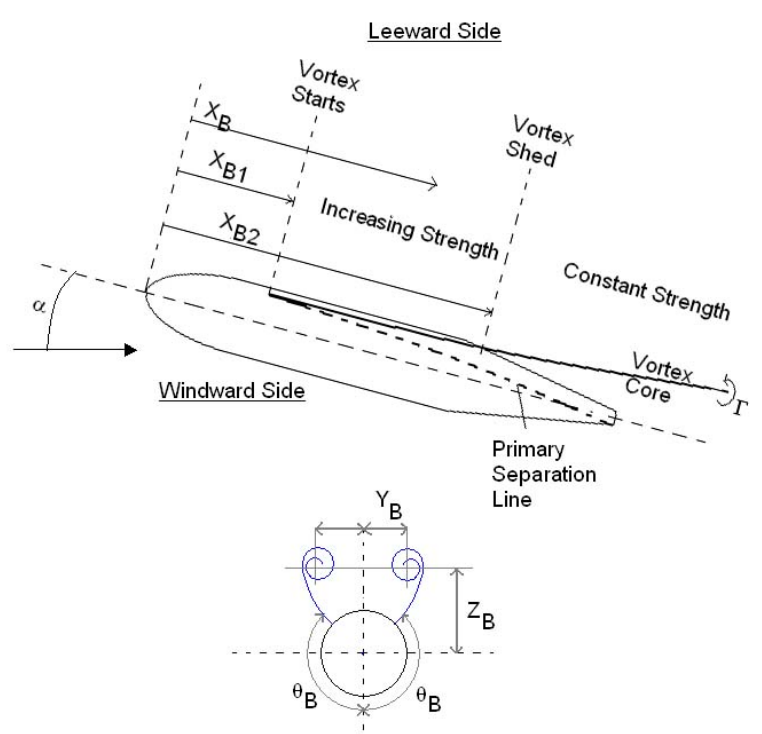

Fig. 1 Body Vortex Notation, adapted from [2]

Secondly, at intermediate incidence angles the crossflow boundary layer separates due to an adverse pressure gradient, $\left(\frac{d P}{d \theta}>0\right)$ on the leeward side. Vorticity shed from the boundary layer is convected away and coalesces to form a steady symmetric body vortex pair on the leeside of the hull. Crossflow separation initiates towards the stern, as the incidence angle increases the separation line moves forwards and leeward, see Figure 1. There is a non-linear increase in lift with incidence angle, due to the low pressure associated with the core of the body vortices which is impressed upon the nearby body surface. At higher angles of attack secondary vortices may form in the stern of the body.

Thirdly, for large incidence angles the body vortices become asymmetric, resulting in a transverse force. Finally, for very large incidence angles the crossflow dominates, the flow pattern tends towards that of the flow around a cylinder, where the boundary 
layer is shed in the form of a Von Karman or random wake.

For an underwater vehicle undergoing a tight turn, the hull experiences intermediate incidence angles and consequently understanding the flow regime and global forces and moments is important if the behaviour of the vehicle is to be correctly modelled. The need to achieve this at the design stage has lead to significant interest in the use of computational fluid dynamics (CFD) to predict the global loads acting on a manoeuvring underwater vehicle.

Good capture of the vortical flow structure in a Reynolds Averaged Navier Stokes (RANS) based computation relies on two key factors. Firstly simulations require a well designed mesh: poor spatial resolution of the vortex results in diffused vortices which decay artificially fast. Secondly an appropriate turbulence closure model (TCM) is required, which can model the influence of turbulence on the flow. It is believed that the discrepancies between numerical and experimentally calculated hydrodynamic derivatives, up to $20 \%$, for a manoeuvring underwater vehicle, observed in previous studies, [3], [4], [5] and [6] are largely due to these issues.

Due to the availability of experimental data detailing crossflow separation several authors have used CFD simulations to model the flow around a prolate spheroid. Gee et al. [7] examined the flow at $\alpha=27^{\circ}$ at a diameter based Reynolds number of $1.1 \times 10^{6}$ using the Baldwin Lomax and Johnson-King turbulence closure models. Comparison between numerical and experimental results highlights the difficulty in correct prediction of the crossflow separation line, and highlights differences in predicted body vortex strength between turbulence models, however these are not compared with experimental results.

Constantinescu et al. [8] compare Detached Eddy Simulation results for the flow around a prolate spheroid with experimental and RANS simulations using the standard and modified versions of the Spalart-Allmaras TCM. In general the flow field predictions of the mean properties of the flow field are similar for the RANS and DES results, however the DES results come with significantly increased computational cost.

Kim et al. [9] used the commercial finite volume CFD code Fluent to model the flow around a prolate spheroid at $\alpha=10^{\circ}, 20^{\circ}$ and $30^{\circ}$. The numerical results for various commonly used turbulence models and modified variants their of, are compared with the experimental data in terms of crossflow separation pattern, static pressure, skin-friction, and wallshear angles on the body surface, and variation of lift and pitching moment with incidence angle. The prediction accuracy varies widely depending on the turbulence model employed. The modified Reynolds stress transport models showed very good improvement in predicting the surface pressure distribution and global loads compared to two equation models. The structure and strength of the vortex is not considered.
Clarke et al. [10] compared numerical and experimental surface pressure and streamlines for a $3: 1$ prolate spheroid at $\alpha=10^{\circ}$ at Reynolds numbers between $0.4 . \times 10^{6}$ to $4.0 \times 10^{6}$. The RANS simulations were performed using the commercial code Fluent and the realisable $k-\varepsilon$ and Shear Stress Transport turbulence closure models with and without transition modelling. Reasonable agreement was found between the surface pressure and streamlines, however the transition modelling failed to result in an improvement in calculation of surface pressures.

In order to understand the ability of turbulence closure models to replicate the structure and path of body vortices as well as correctly predicting crossflow separation, this work replicates the experiments of Lloyd and Campbell [2] on a generic submarine body at 15 degrees incidence numerically. Simulations are based on a RANS approach with five TCMs. Computations are made for steady state fully turbulent flow. By keeping the flow conditions, flow solver and mesh consistent the variations in flow field are attributed to the TCM and wall modelling approaches.

\section{Numerical Model}

The motion of the fluid is modelled using the incompressible (1), isothermal Reynolds Averaged Navier Stokes (RANS) equations (2) in order to determine the mean cartesian flow field, $\overline{U_{i}}$, and the mean pressure $(\mathrm{P})$ of the water around the hull:

$$
\frac{\partial \overline{U_{i}}}{\partial x_{i}}=0
$$

$$
\begin{aligned}
\rho \frac{\partial \overline{U_{i}}}{\partial t}+\rho \frac{\partial \overline{U_{i} U_{j}}}{\partial x_{j}}= & -\frac{\partial P}{\partial x_{i}}+\frac{\partial}{\partial x_{j}}\left\{\mu\left(\frac{\partial \overline{U_{i}}}{\partial x_{j}}+\frac{\partial \overline{U_{j}}}{\partial x_{i}}\right)\right\} \\
& -\rho \frac{\partial \overline{u_{i}^{\prime} u_{j}^{\prime}}}{\partial x_{j}}+f_{i}
\end{aligned}
$$

where $f_{i}$ represents external forces. The influence of turbulence on the mean flow is represented in equation (2) by the Reynolds stress tensor $\left(\rho \overline{u_{i}^{\prime} u_{j}^{\prime}}\right)$.

\subsection{Turbulence Closure}

The four RANS equations have 10 unknowns: three velocities one pressure and nine components (six unique) of the Reynolds stress tensor and thus the equation set is not closed. Various turbulence closure models (TCM) have been proposed to provide solutions to the Reynolds stresses to allow closure.

The models most commonly used in engineering applications are based on the Boussinesq assumption that there exists an analogy between the action of the viscous stresses and the Reynolds stresses on the mean flow. It is assumed that turbulence increases the effective viscosity from $\mu$ to $\mu+\mu_{T}$, where $\mu_{T}$ is 
the eddy viscosity. Hence, the Reynolds stresses can be represented as:

$$
-\rho \overline{u_{i}^{\prime} u_{j}^{\prime}}=\mu_{T}\left(\frac{\partial \overline{U_{i}}}{\partial x_{j}}+\frac{\partial \overline{U_{j}}}{\partial x_{i}}\right)-\rho \frac{2}{3} k \delta_{i j},
$$

where $\delta_{i j}$ is the Kronecker delta $\left(\delta_{i j}=1\right.$ if $i=j$ and $\delta_{i j}=0$ if $i \neq j$ ). The first term on the right hand side is analogous to the viscous stresses, where the molecular viscosity $\mu$ is replaced with the eddy viscosity. The second term ensures the correct result for the normal Reynolds stresses. Equation 3 is known as the isotropic eddy viscosity model since it assumes the ratio between the Reynolds Stresses and mean rate of deformation is the same in all normal directions and consequently assumes that the principal axes of the Reynolds stress tensor are coincident with those of the mean strain rate at all points in the flow. Eddy viscosity TCM seek to find $\mu_{T}$ as a function of the mean flow properties.

Five turbulence models are used for this study: four eddy viscosity models and one second order closure model, where transport equations are directly solved for the Reynolds stresses.

\subsubsection{Spalart-Allmaras TCM}

The Spalart-Allmaras model [11] is a one equation model based upon a transport equation for a viscosity like variable $\tilde{\nu}$. Its formulation and coefficients were defined using dimensional analysis and selected empirical results from 2D mixing layers, wakes and flatplate boundary layers. It has been shown to give reasonably good predictions of $2 \mathrm{D}$ mixing layers, wake flows and flat boundary layers, showing improvements in the prediction of flows with an adverse pressure gradient compared with the $k-\varepsilon$ and $k-\omega \bmod -$ els, [12]. Typical applications of this turbulence model are, [13]; Airplane and wing flows, external automobile aerodynamics, flow around ships. In its original form, the Spalart-Allmaras model is effectively a low-Reynolds-number model, requiring the viscousaffected region of the boundary layer to be properly resolved. For this study the Edwards-Chandra [14] modification is used for the velocity gradient in the turbulence production term.

\subsection{2 $k-\varepsilon T C M$}

The $k-\varepsilon$ model is the TCM which has historically been most commonly used for engineering applications, [15]. It is a two-equation TCM in which model transport equations are solved for two variables the turbulent kinetic energy, $k$, and the rate of dissipation of turbulent energy $\left(\varepsilon=\nu \frac{\overline{\partial u_{i}^{\prime}} \partial \frac{\partial u_{i}^{\prime}}{\partial x_{j}}}{\partial x_{j}}\right.$. These are combined to predict the eddy viscosity, $\nu_{T}$.

\section{$2.1 .3 k-\omega T C M$}

The $k-\omega$ model , [16], is a two-equation TCM in which model transport equations are solved for $(k=$ $\left.\frac{1}{2} \overline{u_{i}^{\prime} u_{i}^{\prime}}\right)$ and the specific dissipation $(\omega=\varepsilon / k)$. Through dimensional analysis these are combined to predict the eddy viscosity; -

The $k-\omega$ model performs well close to walls in boundary layer flows but is known to be sensitive to freestream value of $\omega$.

\subsubsection{SST TCM}

The Shear Stress Transport (SST) model [17] is a two zone model that blends a variant of the $k-\omega$ model in the inner boundary layer with a transformed version of the $k-\varepsilon$ in the outer boundary layer and away from the wall.

Previous investigations for ship flows have shown it is better able to replicate the flow around ship hull forms than either zero equation models or the $k-\varepsilon$ model, notably in capturing hooks in the wake contours at the propeller plane, $[18 ; 19]$.

\subsubsection{SSG Reynolds Stress TCM}

The isotropic turbulent viscosity assumption may lead to inaccurate predictions in complex flows, noteworthy examples are [16]: flows with sudden changes in mean strain rate, flow over curved surfaces, three dimensional flows and flows with boundary layer separation. To overcome the limitations of the Boussinesq assumption Reynolds stress models (RSM) have been proposed which solve six additional transport equations for the unique Reynolds stresses, since turbulence dissipation appears in the individual stress equations, an equation for $\varepsilon$ is still required.

The closure coefficients proposed by Speziale Sarkar and Gatski (SSG) [20] are used in this work. Closure of the equation set still relies on empirical formulations and thus applications of these approaches have shown that they do not always provide better solutions than conventional two-equation models [13].

\subsection{Numerical Implementation}

The RANS equations are implemented in the commercial CFD code ANSYS CFX 11 (CFX), [13]. The governing equations are discretized using the finite volume method. The high-resolution advection scheme was applied. For a scalar quantity, $\phi$ the advection scheme is written in the form

$\phi_{i p}=\phi_{u p}+b \nabla \phi R$

where $\phi_{i p}$ is the value at the integration point, $\phi_{u p}$ is the value at the upwind node and $R$ is the vector from the upwind node to the integration point. The model is first order when $b=0$ and is a second order upwind biased scheme for $b=1$. The high resolution scheme calculates $b$ using a similar approach as that given by [21], which aims to maintain $b$ locally to be as close to one as possible without introducing local oscillations. Collocated (non-staggered) grids are used for all transport equations, and pressure velocity coupling is achieved using an interpolation scheme based 
on that proposed by [22]. Gradients are computed at integration points using tri-linear shape functions defined in [13]. The linear set of equations that arise by applying the Finite Volume Method to all elements in the domain are discrete conservation equations. The system of equations is solved using a fully coupled solver and a multigrid approach [13].

\section{Generic Submarine Body (DOR) at $\alpha=15^{\circ}$ Incidence}

Lloyd and Campbell [2] performed towing tank and rotating arm experiments on a $5 \mathrm{~m}$ long representative submarine body, designation DOR of the UK, Admiralty Research Establishment. The axi-symmetric body comprises an ellipsoid bow moving to parallel midbody with a fineness ratio (length/diameter) of 8.5, with moves to a tapered stern section, see Figure 2. Tests where performed at a diameter based Reynolds number of $1.3 \times 10^{6}$, which corresponds to a length based Reynolds number of $11 \times 10^{6}$. The vorticity surrounding the body was measured using a Freestone vorticity probe [23]. The strength and location of the vortex structures are presented. The measurements of the vortex structure is the reason that this body was studied rather than the more commonly considered DARPA SUBOFF.

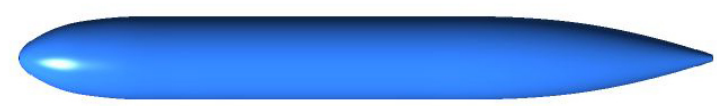

Fig. 2 DOR Body

Table 1 Ordinates of DOR Body, $L=5 m$ and $R_{\max }=$ $0.294 m$.

\begin{tabular}{ll}
\hline$X_{B} / L$ & $R / R_{\max }$ \\
\hline 0.00 & 0.000 \\
0.05 & 0.699 \\
0.10 & 0.905 \\
0.15 & 0.988 \\
0.20 & 1.000 \\
0.25 & 1.000 \\
0.30 & 1.000 \\
0.35 & 1.000 \\
0.40 & 1.000 \\
0.45 & 1.000 \\
0.50 & 1.000 \\
0.55 & 1.000 \\
0.60 & 1.000 \\
0.65 & 1.000 \\
0.70 & 0.983 \\
0.75 & 0.932 \\
0.80 & 0.840 \\
0.85 & 0.701 \\
0.90 & 0.513 \\
0.95 & 0.286 \\
1.00 & 0.000 \\
\hline
\end{tabular}

To illustrate the influence of mesh density on vortex resolution a mesh sensitivity study was performed on a series of five structured meshes, systematically refined over the whole domain. Since uncertainty in the solution can also be attributed to the turbulence model, five turbulence models will be used.

1. Spalart-Allmaras (SP-A) with Edwards-Chandra modification - Default (low Re Formulation)

2. Standard $k-\varepsilon$ - Scalable Wall Functions

3. Standard $k-\omega-$ Automatic Wall Functions (Mixed formulation)

4. Shear Stress Transport (SST) - Automatic Wall Functions (Mixed formulation)

5. SSG Reynolds Stress Model (SSG RSM) - Scalable Wall Functions

It is assumed that the flow is fully turbulent, since two previous studies examining the flow around prolate spheroids at similar Reynolds numbers $[9 ; 10]$, observed no significant improvements when considering laminar flow and transition near the bow.

\subsection{Domain and Mesh}
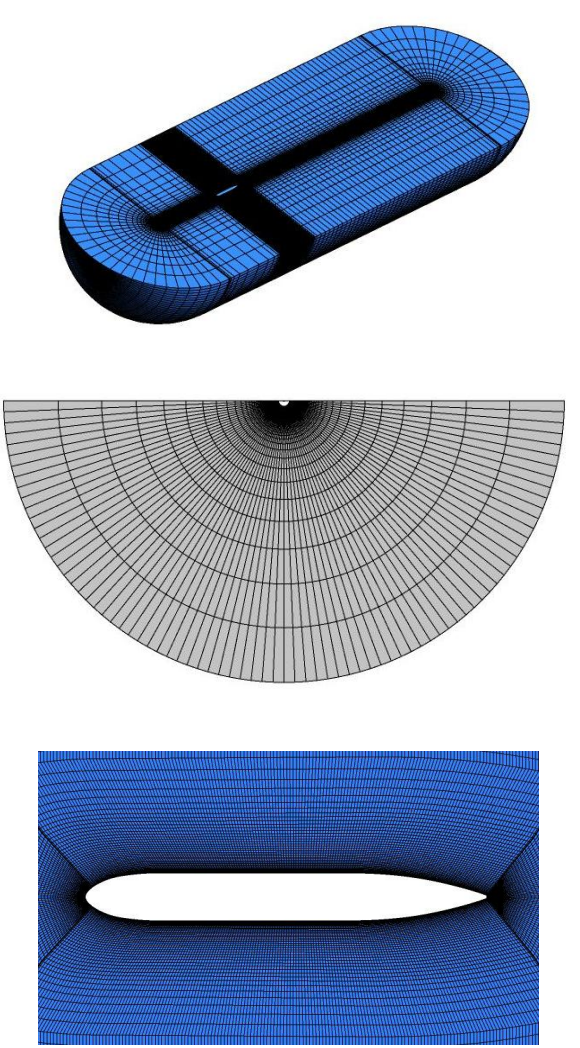

Fig. 3 Mesh total domain (top), mesh cut plane $X_{B} / L=0.5$ (middle) and mesh cut plane x-z (bottom). Note: for clarity the medium mesh is presented

The computational domain is illustrated in Figure 3. It extends $6 \mathrm{~L}$ upstream, $11 \mathrm{~L}$ downstream and $3 \mathrm{~L}$ transversely, half the geometry is modelled explicitly, the rest is considered by applying a symmetry condition. The final $1 \%$ of the body length is truncated to ensure good quality elements at the stern of the body.

Structured meshes are built using the commercial meshing package ANSYS ICEM CFD V11 (ICEM). A 
triple 0-grid topology is applied surrounding the hull, the inner zone extending to twice the trailing edge boundary layer thickness, $R=2 \delta$, is designed to capture the boundary layer, the middle zone extending out to $R=1 D$ is used to provide a fine mesh density to resolve body vortices, the final zone extending out a further $1 D, R=2 D$, is used to allow rapid expansion from the near to farfield mesh regimes. An initial fine mesh is built first. This is then coarsened using a mesh reduction ratio of $1 / \sqrt{2}$ on each edge to produce the series of progressively coarser meshes. Mesh sizes are matched at all zone boundaries. The mesh coarsening process was applied automatically in ICEM, while matching of the mesh boundaries was performed by hand. The fist layer thickness was adjusted based on the number of nodes along each edge.

Maintaining a constant $y^{+}$value over a three dimensional body experiencing significant flow separation such as a submarine at a large drift angle is not feasible. For this study the first cell thickness is selected to give the appropriate $y^{+}$over the attached region of flow. This results in a significantly lower $y^{+}$ value in the detached regions.

To investigate the influence of wall function approaches two sets of meshes are built. These meshes have identical topology, but the difference arises in the edge distributions used in the three concentric ' $\mathrm{O}$ ' grids surrounding the body. The first set comprises five meshes designed to use wall functions where the finest has a nominal $y^{+}$value of 30 with 30 elements in the boundary layer, the nominal $y^{+}$is taken to be the value experienced over the majority of the hull experiencing attached flow. It typically has a maximum of 50 near the bow and reduces to 10 within the separated zone. Even on the coarsest mesh $y^{+}$is never more than 200. In the case of scalable wall functions, the formulation is sufficiently robust that the singularity of the numerical method is not experienced.

The second series of three meshes is designed to integrate the flow down to the wall with the finest mesh having a nominal $y^{+} \sim 1$ with 30 elements in the boundary layer. For both sets of meshes the total number of elements in the boundary layer was kept constant to ensure both sets had identical topologies and numbers of elements. Consequently, the resolution of the with wall function mesh is finer away from the wall than the $y^{+}=1$ mesh, which may have a small influence on the results.

Initially the fist cell thickness, $\Delta y$ required was estimated using the following empirical equation, [13]:

$\Delta y=L \Delta y^{+} \sqrt{80} R e^{-13 / 14}$

After initial simulations the first layer thickness was tuned to ensure that it matched the desired value.

The finest mesh comprises of $10.3 \mathrm{M}$ elements, $46 \%$ of the elements in the boundary layer O-Grid, $41 \%$ in the second O-grid for capturing the body vortices and the final $13 \%$ of the elements is used to discretize the farfield.
Three boundary conditions are applied to the simulation. A no slip wall condition on the DOR body a reflection plane on the $\mathrm{x}-\mathrm{z}$ plane and an opening on the farfield boundary. The opening has a flow velocity of $2 \mathrm{~m} / \mathrm{s}$, the inflow has a $5 \%$ turbulence intensity.

The simulation co-ordinate system is aligned with DOR body, see Figure 1, not with the flow direction since this gives a first order estimate of the body vortex aligned axis system. Measured drag, lift and pitch moment are presented with respect to the flow direction. Pitch moment is calculated around amidships.

\subsection{Computation}

Simulations were performed using the University of Southampton's Iridis 2 Beowulf cluster, based on AMD Opteron processors, running RedHat Enterprise Linux. Typical run times and computational resources are described in Table 2. The one and two equation models were initiated assuming a uniform freestream velocity. The SSG RSM was initiated using the converged solution from the SST run. Simulations are continued until the normalised RMS of all residuals are less than $10^{-5}$. A reduction of three orders of magnitude. No significant variation in the path and strength of the vortex or the magnitude of the global forces was observed once the RMS residuals had reduced below $5 \times 10^{-5}$.

For these steady state simulations the CFX-Solver applies a pseudo timestep as a method of under-relaxing the equations as they iterate towards the final solution. As the solver is fully implicit, a relatively large time scale can typically be selected, so that the convergence to steady-state is as fast as possible. For these studies a pseudo timestep of 0.1 s was used.

\subsection{Grid Uncertainty Analysis}

The procedures and methodology used are based upon those presented in [24], based on an Richardson extrapolation [25].

Table 3 shows the global integrated forces on the $y^{+} \sim 30$ meshes. As already noted the first cell thickness is set to give the desired nominal $y^{+}$in the attached crossflow region. In the separated zone and small stagnation region the local $y^{+}$drops below the desired value. For the automatic wall formulations used by the SST and $k-\omega$ model they switch between a wall function and low-Re approach depending on the local $y^{+}$. For the VF-y30 mesh the local $y^{+}$ drops below 11 over some of the separated zone. This changes how the near wall flow variables are calculated and this may account for the larger grid uncertainty associated with these two cases. The $k-\varepsilon$ and SSG RSM which use scalable wall functions demonstrate improved mesh convergence.

Table 4 compares the global hydrodynamic forces acting on the DOR body at an incidence angle of $15^{\circ}$ on the $y^{+}=1$ meshes. For the low Re wall modelling 
Table 2 Computational Resources and run times (Note: runtime for SSG RSM is time taken to reach a converged solution based on the initially converged results for the SST TCM)

\begin{tabular}{|c|c|c|c|c|c|}
\hline TCM & Mesh & $\begin{array}{l}\text { No. } \\
\text { Processors }\end{array}$ & $\begin{array}{l}\text { Total } \\
\text { Memory }\end{array}$ & $\begin{array}{l}\text { No. } \\
\text { Iterations }\end{array}$ & $\begin{array}{l}\text { Total Wall } \\
\text { Clock Time }\end{array}$ \\
\hline SP-A & M-y1 & 1 & $2 \mathrm{~Gb}$ & 205 & 10hr $39 \mathrm{~min}$ \\
\hline SP-A & F-y1 & 16 & $16 \mathrm{~Gb}$ & 200 & $2 \mathrm{hr} 58 \mathrm{~min}$ \\
\hline SP-A & VF-y1 & 16 & $32 \mathrm{~Gb}$ & 149 & $5 \mathrm{hr} 59 \mathrm{~min}$ \\
\hline$k-\varepsilon$ & VC-y30 & 1 & $2 \mathrm{~Gb}$ & 180 & $1 \mathrm{hr} 22 \mathrm{~min}$ \\
\hline$k-\varepsilon$ & С-y30 & 1 & $2 \mathrm{~Gb}$ & 166 & $3 \mathrm{hr} 22 \mathrm{~min}$ \\
\hline$k-\varepsilon$ & M-y30 & 1 & $2 \mathrm{~Gb}$ & 151 & $7 \mathrm{hr} 43 \mathrm{~min}$ \\
\hline$k-\varepsilon$ & F-y30 & 16 & $16 \mathrm{~Gb}$ & 139 & $2 \mathrm{hr} 17 \mathrm{~min}$ \\
\hline$k-\varepsilon$ & VF-y30 & 16 & $32 \mathrm{~Gb}$ & 123 & $5 \mathrm{hr} 15 \mathrm{~min}$ \\
\hline$k-\omega$ & VC-y30 & 1 & $2 \mathrm{~Gb}$ & 172 & 1h $22 \mathrm{~min}$ \\
\hline$k-\omega$ & C-y30 & 1 & $2 \mathrm{~Gb}$ & 172 & $3 \mathrm{hr} 24 \mathrm{~min}$ \\
\hline$k-\omega$ & M-y30 & 1 & $2 \mathrm{~Gb}$ & 173 & $9 \mathrm{~h} 48 \mathrm{~min}$ \\
\hline$k-\omega$ & F-y30 & 16 & $16 \mathrm{~Gb}$ & 169 & $2 \mathrm{hr} 45 \mathrm{~min}$ \\
\hline$k-\omega$ & VF-y30 & 16 & $32 \mathrm{~Gb}$ & 161 & $6 \mathrm{hr} 53 \mathrm{~min}$ \\
\hline$k-\omega$ & M-y1 & 1 & $2 \mathrm{~Gb}$ & 200 & $11 \mathrm{hr} 13 \mathrm{~min}$ \\
\hline$k-\omega$ & F-y1 & 16 & $16 \mathrm{~Gb}$ & 193 & $3 \mathrm{hr} 7 \mathrm{~min}$ \\
\hline$k-\omega$ & VF-y1 & 16 & $32 \mathrm{~Gb}$ & 189 & $8 \mathrm{hrs} 44 \mathrm{~min}$ \\
\hline $\mathrm{SST}$ & VC-y30 & 1 & $2 \mathrm{~Gb}$ & 160 & $1 \mathrm{hr} 19 \mathrm{~min}$ \\
\hline $\mathrm{SST}$ & С-y30 & 1 & $2 \mathrm{~Gb}$ & 143 & $3 \mathrm{hr} 17 \mathrm{~min}$ \\
\hline $\mathrm{SST}$ & M-y30 & 1 & $2 \mathrm{~Gb}$ & 147 & $8 \mathrm{hr} 14 \mathrm{~min}$ \\
\hline $\mathrm{SST}$ & F-y30 & 16 & $16 \mathrm{~Gb}$ & 138 & $2 \mathrm{hr} 18 \mathrm{~min}$ \\
\hline $\mathrm{SST}$ & VF-y30 & 16 & $32 \mathrm{~Gb}$ & 132 & $5 \mathrm{hr} 52 \mathrm{~min}$ \\
\hline $\mathrm{SST}$ & M-y1 & 1 & $2 \mathrm{~Gb}$ & 170 & $10 \mathrm{~h} 3 \mathrm{~min}$ \\
\hline $\mathrm{SST}$ & F-y1 & 16 & $16 \mathrm{~Gb}$ & 158 & $2 \mathrm{hr} 40 \mathrm{~min}$ \\
\hline $\mathrm{SST}$ & VF-y1 & 16 & $32 \mathrm{~Gb}$ & 147 & $6 \mathrm{hr} 24 \mathrm{~min}$ \\
\hline SSG RSM & VC-y30 & 1 & $2 \mathrm{~Gb}$ & 200 & $3 \mathrm{hr} 27 \mathrm{~min}$ \\
\hline SSG RSM & C-y30 & 1 & $2 \mathrm{~Gb}$ & 200 & $6 \mathrm{hr} 44 \mathrm{~min}$ \\
\hline SSG RSM & M-y30 & 1 & $2 \mathrm{~Gb}$ & 338 & $17 \mathrm{hr} 24 \mathrm{~min}$ \\
\hline SSG RSM & F-y30 & 16 & $16 \mathrm{~Gb}$ & 338 & $5 \mathrm{hr} 2 \mathrm{~min}$ \\
\hline SSG RSM & VF-y30 & 16 & $32 \mathrm{~Gb}$ & 323 & $8 \mathrm{hr} 17 \mathrm{~min}$ \\
\hline
\end{tabular}

$(\mathrm{VC}=$ Very Coarse, $\mathrm{C}=$ Coarse, $\mathrm{M}=$ Medium, $\mathrm{F}=$ Fine, $\mathrm{VF}=$ Very Fine)

cases the results show a very good level of mesh convergence.

For both sets of meshes the mesh convergence is limited only to the near field flow as downstream the mesh resolution in the wake is insufficient to maintain the wake structure as it advects. Supplementary studies indicated that fine capture of the downstream wake had little effect on the integrated forces and moments or on the nearbody structure of the vortex.

Figure 4 illustrates the influence of mesh density on wall shear streamlines for the SST case. Separation is indicated by the convergence of skin friction lines. With increasing mesh density the separation line moves forward and to windward, increasing the size of the separated zone which in turn leads to a stronger vortex, increasing lift and drag and a reduction in pitching moment.

Figure 5 compares the vorticity contours at $X_{B} / L=$ 0.925 for the SSG RSM model over the 5 meshes. The influence of the mesh is clearly evident in the shape of the contours of the coarsest mesh, while the three finest meshes show negligible variation in the vorticity plot. The centre of the vortex moves further to windward with increasing mesh density.

It is worth noting the resultant $U_{G}$ is mostly very small for the cases without wall functions. There will be a limited amount of deviation from the ideal geometric uncertainty due to the practicalities associated with the mesh generator used. Related investigations into such effects can be found in 3rd Workshop on CFD Uncertainty Analysis [26]. Overall, using an appropriate measure such as grid uncertainty $U_{G}$ helps clarify the influence of mesh on accuracy. It still does not provide a fully satisfactory approach to identifying, even for this relatively simple mesh topology, the relative influence of different TCM. 


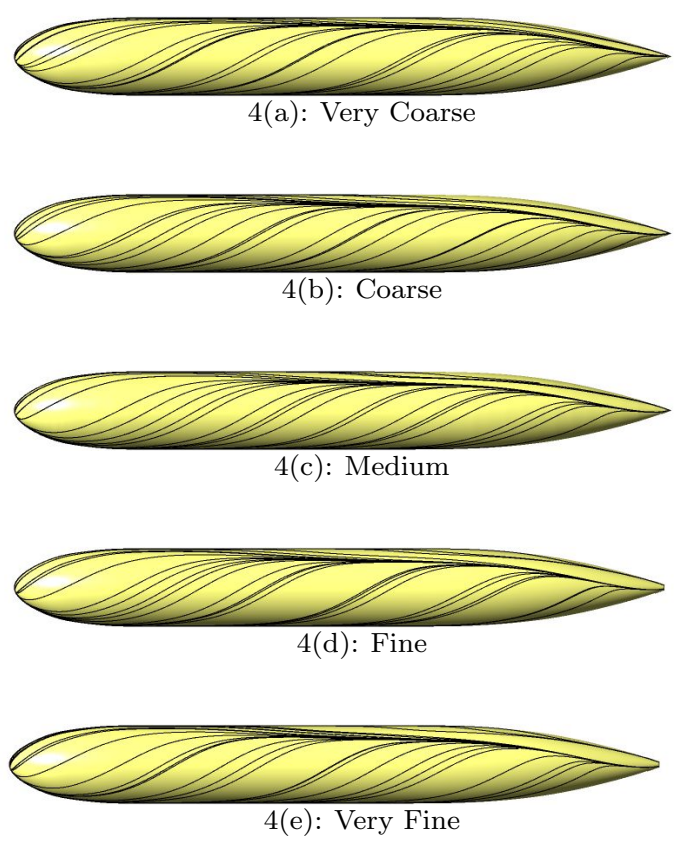

Fig. 4 Surface wall shear streamlines for SST $y^{+} \approx 30$. Note: windward side is the bottom of each figure, leeward side is the top.
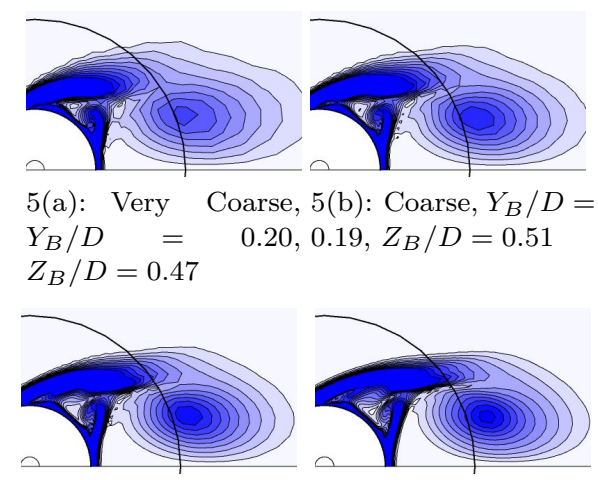
5(c): $\quad$ Medium, $5(\mathrm{~d}):$ Fine, $Y_{B} / D=$ $Y_{B} / D=0.18,0.18, Z_{B} / D=0.52$
$Z_{B} / D=0.51$
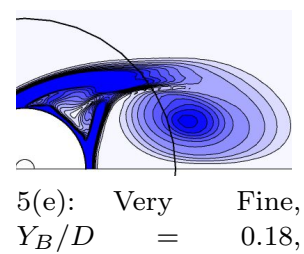

$Z_{B} / D=0.52$

Fig. 5 Vorticity contours in rectilinear flow, $\alpha=15^{\circ}, X_{B} / L=$ 0.925 , SSG RSM for 5 meshes. The Thin Black line represents maximum body diameter, Thick black line represents local body diameter. 
Table 3 Global Integrated Forces acting on the DOR body at $15^{\circ}$ incidence - Wall Functions.

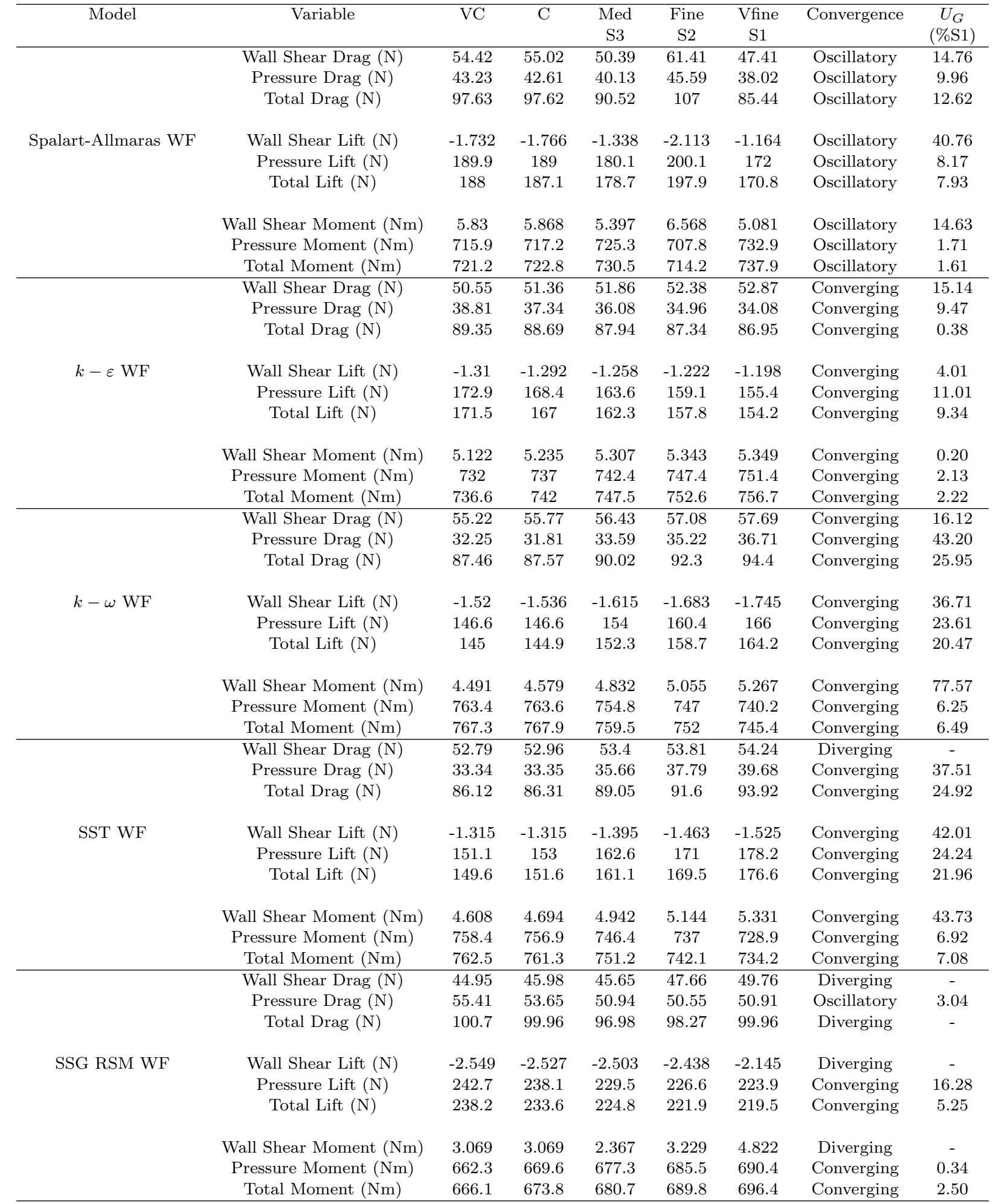


Table 4 Global Integrated Forces acting on the DOR body at $15^{\circ}$ incidence - no wall functions $\left(y^{+} \sim 1\right)$

\begin{tabular}{|c|c|c|c|c|c|c|c|c|}
\hline Model & Variable & $\mathrm{VC}$ & $\mathrm{C}$ & $\begin{array}{c}\text { Med } \\
\text { S3 }\end{array}$ & $\begin{array}{c}\text { Fine } \\
\text { S2 }\end{array}$ & $\begin{array}{c}\text { Vfine } \\
\text { S1 }\end{array}$ & Convergence & $\begin{array}{c}U_{G} \\
(\% \mathrm{~S} 1)\end{array}$ \\
\hline & Wall Shear Drag $(\mathrm{N})$ & - & - & 36.41 & 35.57 & 35.27 & Converging & 1.23 \\
\hline & Pressure Drag $(\mathrm{N})$ & - & - & 32.54 & 31.39 & 30.55 & Converging & 7.45 \\
\hline & Total Drag $(\mathrm{N})$ & - & - & 68.95 & 67.19 & 65.3 & Diverging & - \\
\hline \multirow[t]{9}{*}{ Spalart-Allmaras $y^{+} 1$} & Wall Shear Lift $(\mathrm{N})$ & - & - & -0.8309 & -0.7795 & -0.7762 & Converging & 0.82 \\
\hline & Pressure Lift $(\mathrm{N})$ & - & - & 151.6 & 147.3 & 143.9 & Converging & 8.93 \\
\hline & Total Lift $(\mathrm{N})$ & - & - & 150.7 & 146.4 & 143 & Converging & 8.98 \\
\hline & Wall Shear Moment (Nm) & - & - & 3.672 & 3.532 & 3.419 & Converging & 13.83 \\
\hline & Pressure Moment (Nm) & - & - & 753.6 & 758.6 & 762.5 & Converging & 1.81 \\
\hline & Total Moment (Nm) & - & - & 757.1 & 762.1 & 765.8 & Converging & 1.38 \\
\hline & Wall Shear Drag (N) & - & - & 54.83 & 55.09 & 55.46 & Diverging & 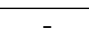 \\
\hline & Pressure Drag $(\mathrm{N})$ & - & - & 39.81 & 39.2 & 38.96 & Converging & 0.83 \\
\hline & Total Drag (N) & - & - & 94.64 & 94.29 & 94.41 & Oscillatory & - \\
\hline \multirow{9}{*}{$k-\omega y^{+} 1$} & Wall Shear Lift (N) & - & - & -1.759 & -1.724 & -1.713 & Converging & 0.99 \\
\hline & Pressure Lift $(\mathrm{N})$ & - & - & 177.8 & 175.4 & 174.4 & Converging & 0.16 \\
\hline & Total Lift $(\mathrm{N})$ & - & - & 176 & 173.7 & 172.6 & Converging & 0.05 \\
\hline & Wall Shear Moment (Nm) & - & - & 5.577 & 5.59 & 5.626 & Diverging & - \\
\hline & Pressure Moment (Nm) & - & - & 725.5 & 728 & 729.1 & Converging & 0.03 \\
\hline & Total Moment (Nm) & - & - & 730.9 & 733.5 & 734.7 & Converging & 0.02 \\
\hline & Wall Shear Drag (N) & - & - & 52.44 & 52.31 & 52.39 & Oscillatory & - \\
\hline & Pressure Drag $(\mathrm{N})$ & - & - & 42.55 & 42.37 & 42.27 & Converging & 0.06 \\
\hline & Total Drag (N) & - & - & 94.99 & 94.67 & 94.66 & Converging & 0.02 \\
\hline \multirow[t]{9}{*}{$\operatorname{SST} y^{+} 1$} & Wall Shear Lift $(\mathrm{N})$ & - & - & -1.626 & -1.57 & -1.532 & Converging & 5.24 \\
\hline & Pressure Lift $(\mathrm{N})$ & - & - & 188.4 & 188 & 187.7 & Converging & 0.48 \\
\hline & Total Lift (N) & - & - & 186.8 & 186.4 & 186.2 & Converging & 0.00 \\
\hline & Wall Shear Moment (Nm) & - & - & 5.675 & 5.668 & 5.686 & Oscillatory & - \\
\hline & Pressure Moment (Nm) & - & - & 715.2 & 716.5 & 717.2 & Converging & 0.02 \\
\hline & Total Moment (Nm) & - & - & 720.7 & 722.1 & 722.8 & Converging & 0.00 \\
\hline & Wall Shear Drag (N) & - & - & 48.14 & 48.72 & 49.03 & Converging & 0.09 \\
\hline & Pressure Drag $(\mathrm{N})$ & - & - & 52.65 & 51.16 & 50.2 & Converging & 1.55 \\
\hline & Total Drag $(\mathrm{N})$ & - & - & 99.71 & 98.75 & 98.09 & Converging & 1.48 \\
\hline \multirow[t]{6}{*}{$\operatorname{SSG} \operatorname{RSM} y^{+} 1$} & Wall Shear Lift (N) & - & - & -1.946 & -1.902 & -1.882 & Converging & 0.18 \\
\hline & Pressure Lift $(\mathrm{N})$ & - & - & 229.2 & 223.5 & 219.9 & Converging & 1.17 \\
\hline & Total Lift $(\mathrm{N})$ & - & - & 224.9 & 219.3 & 215.6 & Converging & 1.63 \\
\hline & Wall Shear Moment (Nm) & _ & - & 5.172 & 5.176 & 5.183 & Diverging & - \\
\hline & Pressure Moment (Nm) & - & - & 683.7 & 690.6 & 694.9 & Converging & 0.40 \\
\hline & Total Moment (Nm) & - & - & 689.2 & 696.3 & 700.7 & Converging & 0.40 \\
\hline
\end{tabular}




\section{Results}

All five turbulence models demonstrate the same general flow field, see Figure 6, for the SSG RSM. The flow stagnates on the windward side of the bow, the flow accelerates over the curvature of the hull, separating on the leeward side due to the adverse pressure gradient. The separated flow leads to two free vortex sheets which roll up to form the pair of body vortices which increase in strength as they flow past the hull. In the stern of the hull secondary vortices start to form to leeward of the primary vortices.

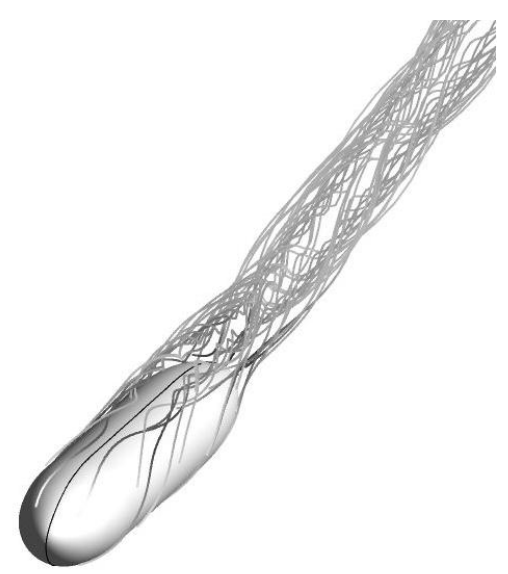

Fig. 6 Cross-flow separation and streamwise vortices on the DOR submarine body at incidence angle of $15^{\circ}$

\subsection{Global Forces}

Figure 7 compares the global loads calculated on the very fine meshes. Selection of TCM has a significant impact on the global forces acting on the hull. The drag varies by $42.0 \%^{1}$, lift varies by $42.2 \%$ and the pitch moment varies by $9.5 \%$. Typically the drag force is $50-65 \%$ skin friction and $35-50 \%$ pressure drag. The lift force and pitching moment are dominated by the pressure component $>99 \%$. A similar study by Kim and Rhee [9] on a 6:1 prolate spheroid showed similar variations in integrated global loading when calculated using different TCMs (SST, $k-\omega$, SpalartAllmaras). At $20^{\circ}$ incidence on a fine mesh without wall functions the lift varied by $45 \%$ and the pitching moment varied by $34 \%$.

Figure 8 shows the hull pressure distribution predicted by the five TCMs. All show good agreement over the majority of the length of the hull, with differences concentrated in the stern leeward region of the hull. Figure 9 shows pressure distribution at $X_{B} / L=$ 0.925 and the difference in hull pressure distribution can be clearly seen. Previous studies have shown that the pressure distribution in the separated zone should be approximately constant. This is observed for all turbulence models in the zone from $90^{\circ}$ to $180^{\circ}$, with the SSG RSM having the lowest pressure in this region.

\footnotetext{
$1((\max -\min ) * 2 /(\max +\min ) * 100)$
}

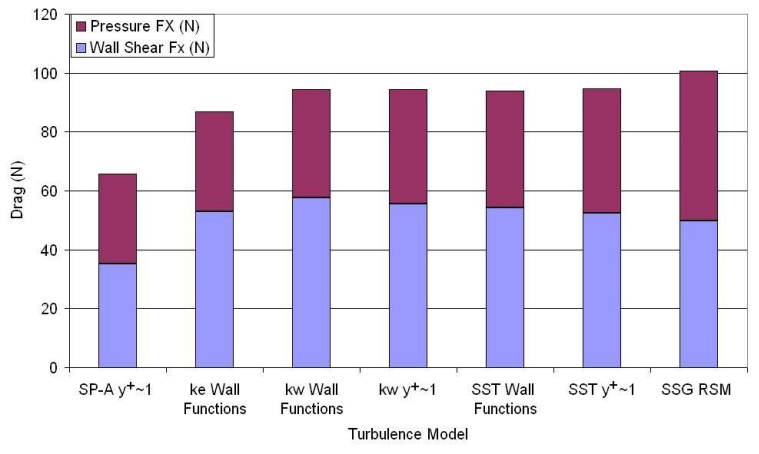

$7(\mathrm{a})$ : drag
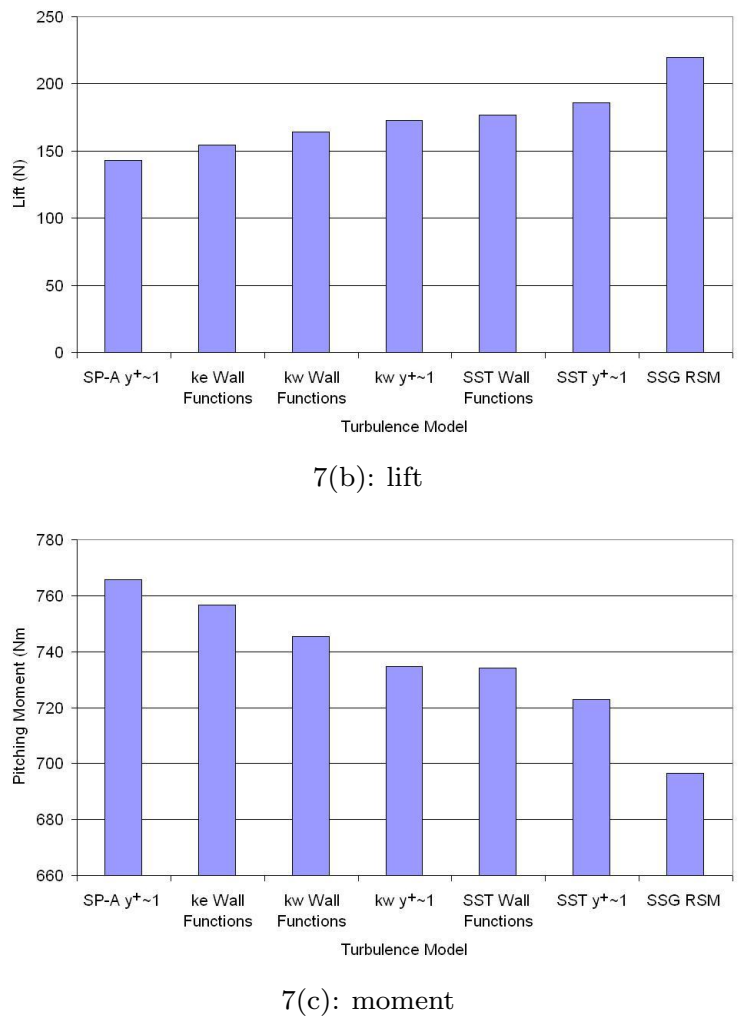

Fig. 7 Comparison of global loads calculated on the finest meshes.

Significant variation is observed in the forces and moments acting on the DOR body calculated from the five TCMs As such, extreme care should always be exercised when selecting a TCM for this type of flow.

\subsection{Structure and Path of the Vortex}

The strength and location of the body vortex in relation to the hull will modify the pressure field around the stern of the body modifying the global forces and moments acting on the body. Experimentally the structure and path of the vortex was extracted using a Freestone probe. The output from this when suitably processed gives a direct measure of the vorticity. Hence it was possible to extract the vortex peak location without mapping the entire flow. Lloyd and Campbell [2] note that because the levels of vorticity are low inaccuracies in the Freestone probe caused 


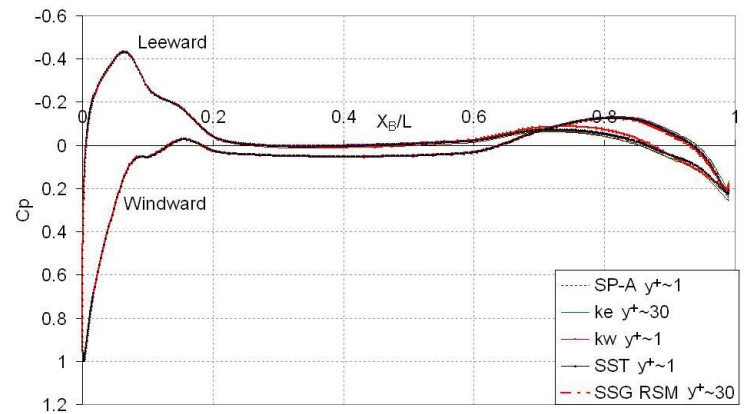

Fig. 8 Longitudinal pressure distribution around hull. $C_{p}=$ $\frac{P-P_{0}}{1 / 2 \rho U^{2}}$.

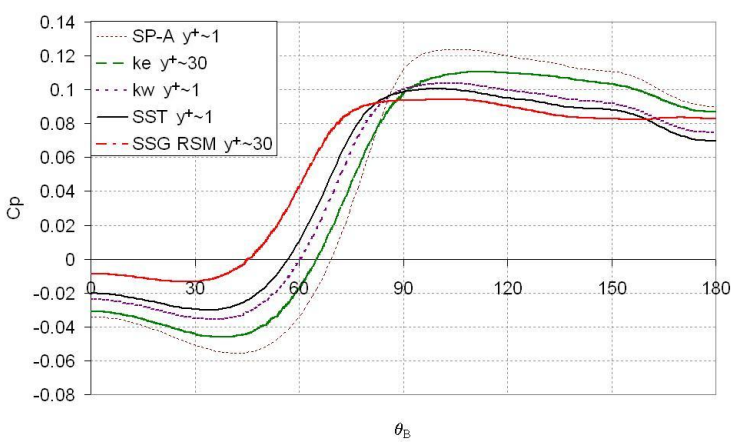

Fig. 9 Pressure distribution around hull at $X_{B} / L=0.925$. $C_{p}=\frac{P-P_{0}}{1 / 2 \rho U^{2}}$

some uncertainty regarding the extent of the vortex and the value of the measured peak.

Figure 10 compares the vorticity contours at $X_{B} / L=$ 0.925 for the experimental and computational models. The experimental results, Figure 10(a), show a strong sheet of vorticity attached to the hull, which is feeding the primary vortex. This is replicated in the results for all five TCMs. However, the peak vorticity varies significantly between the SSG RSM and the other TCMs based upon the Boussinesq approximation. Looking in detail at the structure of the vortex, Figure 11 compares experimental and numerical vorticity for a radial traverse at fixed $\theta_{B}$. The traverse at $180^{\circ}$ highlights a slight asymmetry in the experimental data, this may be either a function of experimental set up or representative of asymmetric vortex production. To investigate this discrepancy a single case (SST VF-y1) was re-analysed modelling the entire domain. No asymmetry was observed in the path or structure of the vortex for this TCM.

The numerical results are forced to a symmetric case by the use of a symmetry boundary condition. The traverses at $160^{\circ}$ and $170^{\circ}$ show that the SSG RSM is closer to replicating the strength of the body vortex than the one and two equation models which under predict the peak vorticity by $50 \%$. However, none of the TCMs well capture the shape of the vorticity transverses.

The path of the vortex core is found using a modified form of the VORTFIND Algorithm, [27] by analysing the transverse flow in body fitted co-ordinates at a series of stations along the body, see Figure 12.

In the $Y_{B}$ direction the vortex tracks from the five TCMs, see Figure 13 are relatively consistent and match quite closely to the vortex probe results. In the $Z_{B}$ direction the predicted vortex tracks from the five TCMs are similar up to $X_{B} / L=0.7$ which corresponds to the limit of the parallel midbody. As the hull tapers at the stern, the experimental results show the vortex core convecting downstream parallel to the hull centreline. However, the vortex tracks from the TCMs follow the curvature of the hull to varying degrees. The track from the SP-A remains approximately equidistant to the hull as it travels downstream while the SSG RSM predicts the track closest to the experimental results. It is possible that the body fitted nature of the mesh structure may be partly responsible for inducing this effect. However, the same phenomena is not experienced in the $Y_{B}$ direction. 


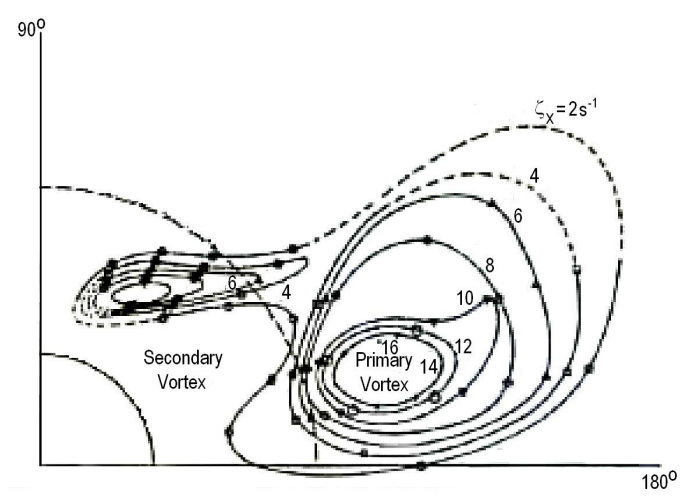

10(a): Experimental Results [2]

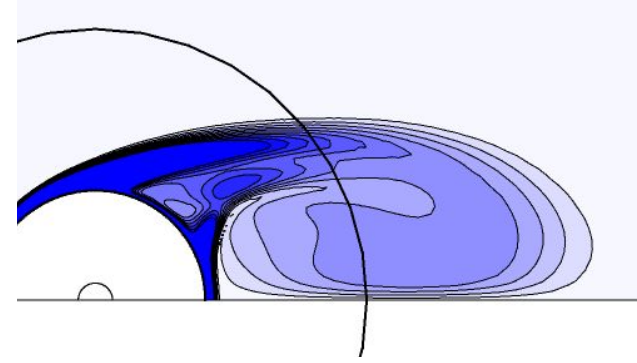

10(c): $k-\varepsilon$

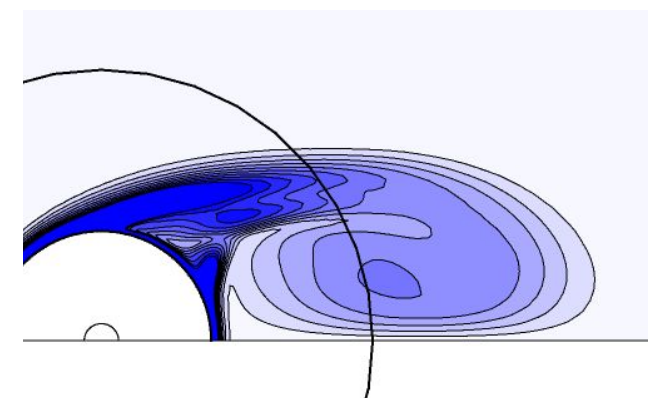

10(e): $k-\omega y^{+} \sim 1$

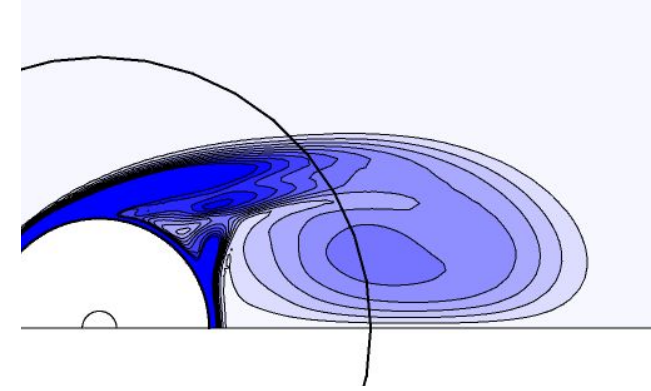

10(g): $\operatorname{SST} y^{+} \sim 1$

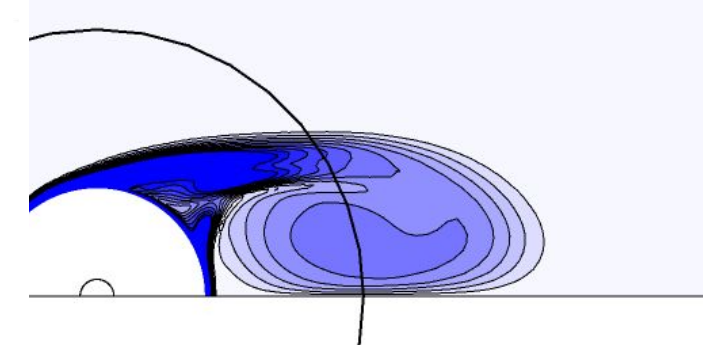

10(b): Spalart-Allmaras

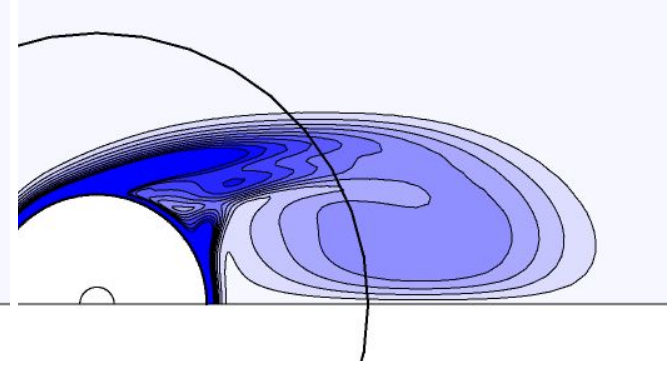

10(d): $k-\omega y^{+} \sim 30$

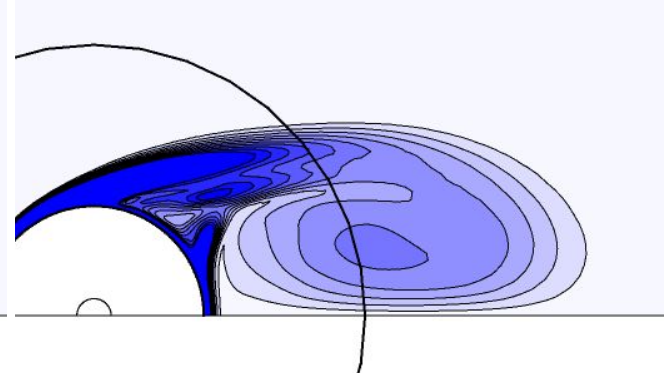

10(f): $\operatorname{SST} y^{+} \sim 30$

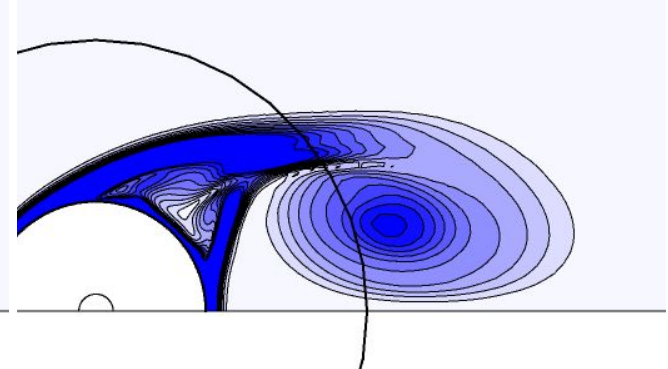

10(h): SSG RSM

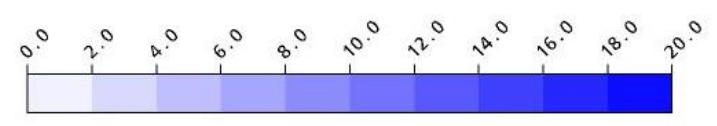

$\left[s^{\wedge}-1\right]$

Fig. 10 Vorticity Contours in Rectilinear Flow, $\alpha=15^{\circ}, X_{B} / L=0.925$ 


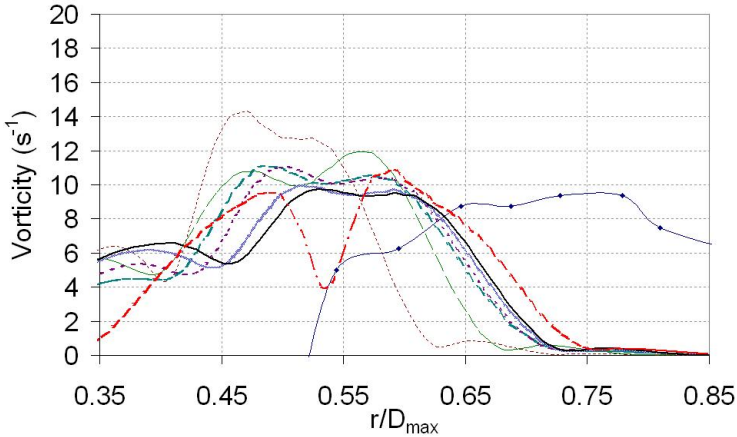

11(a): $\theta_{B}=150^{\circ}$

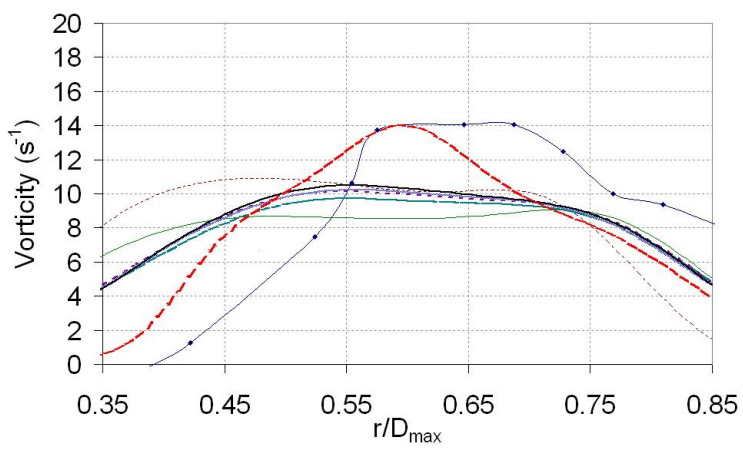

11(c): $\theta_{B}=170^{\circ}$

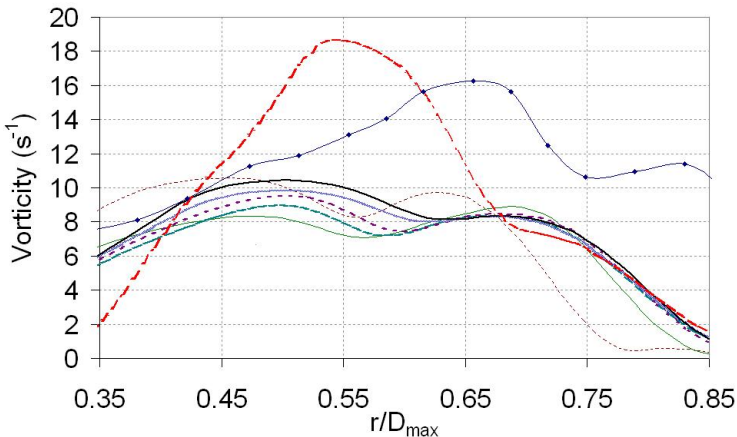

11(b): $\theta_{B}=160^{\circ}$

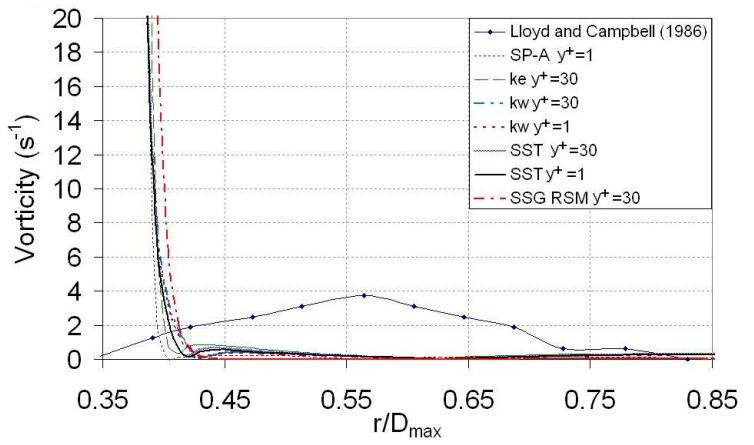

11(d): $\theta_{B}=180^{\circ}$

Fig. 11 Comparison of experimental and numerical vorticity traverses, $X_{B} / L=0.925$ 


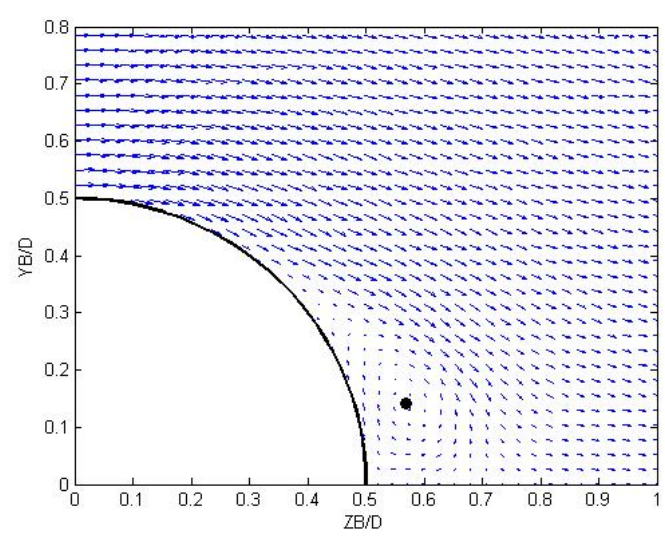

12(a): $k-\varepsilon X_{B} / L=0.5$

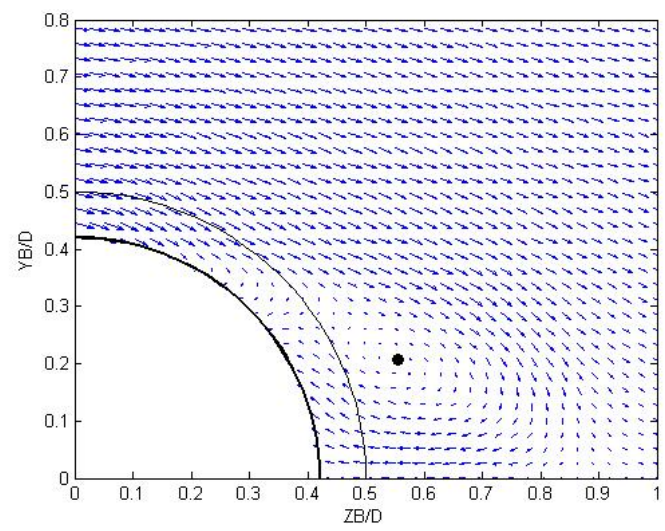

$12(\mathrm{c}): k-\varepsilon X_{B} / L=0.8$

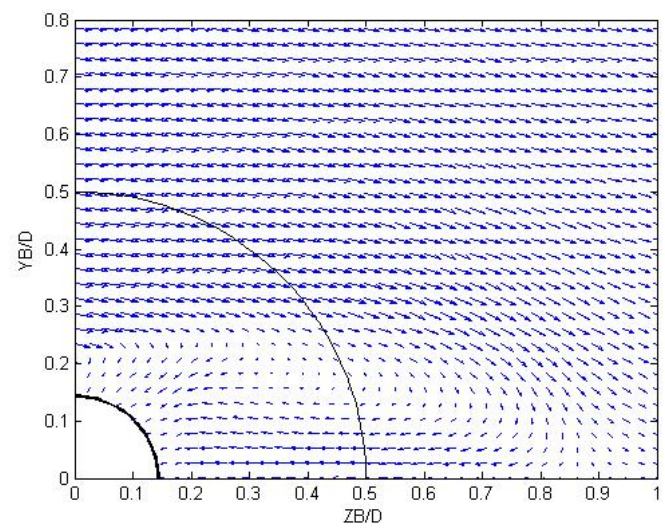

12(e): $k-\varepsilon X_{B} / L=0.925$

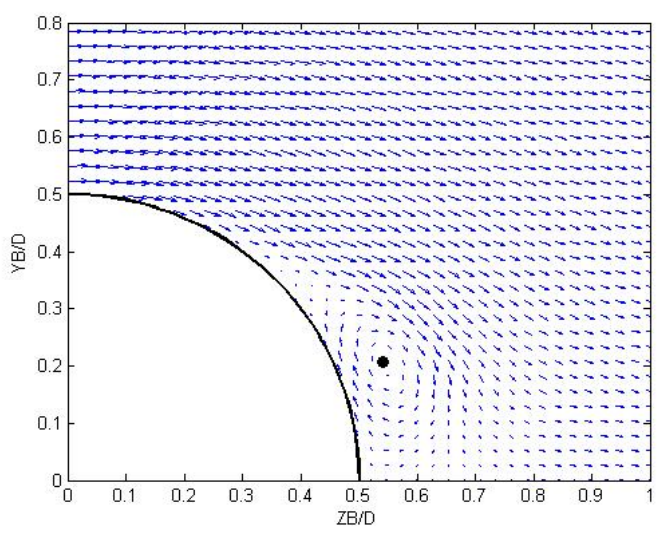

12(b): SSG RSM $X_{B} / L=0.5$

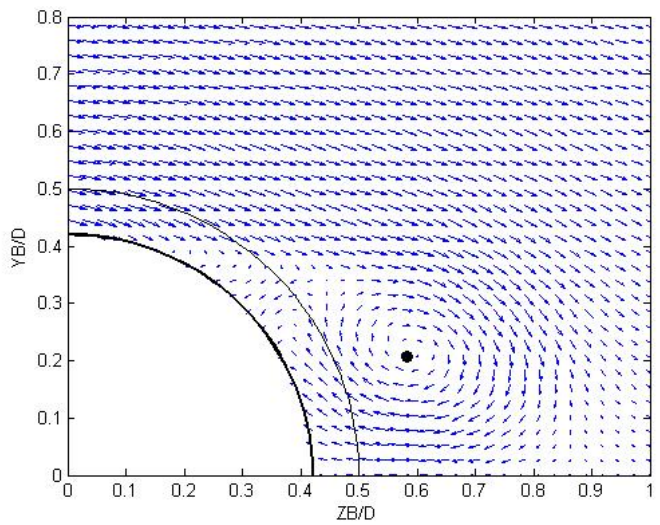

12(d): SSG RSM $X_{B} / L=0.8$

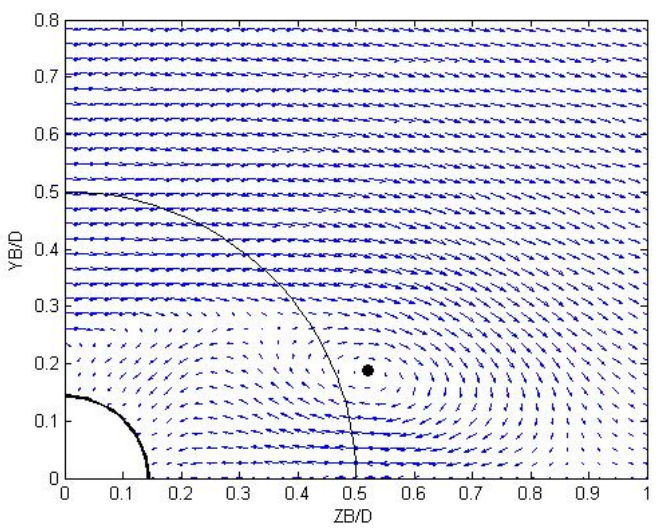

12(f): SSG RSM $X_{B} / L=0.925$

Fig. 12 Transverse velocity vectors at stations along the Body, Thin Black line represents maximum body diameter, Thick black line represents local body diameter. Black spot marks vortex centre located by modified VORTFIND algorithm 

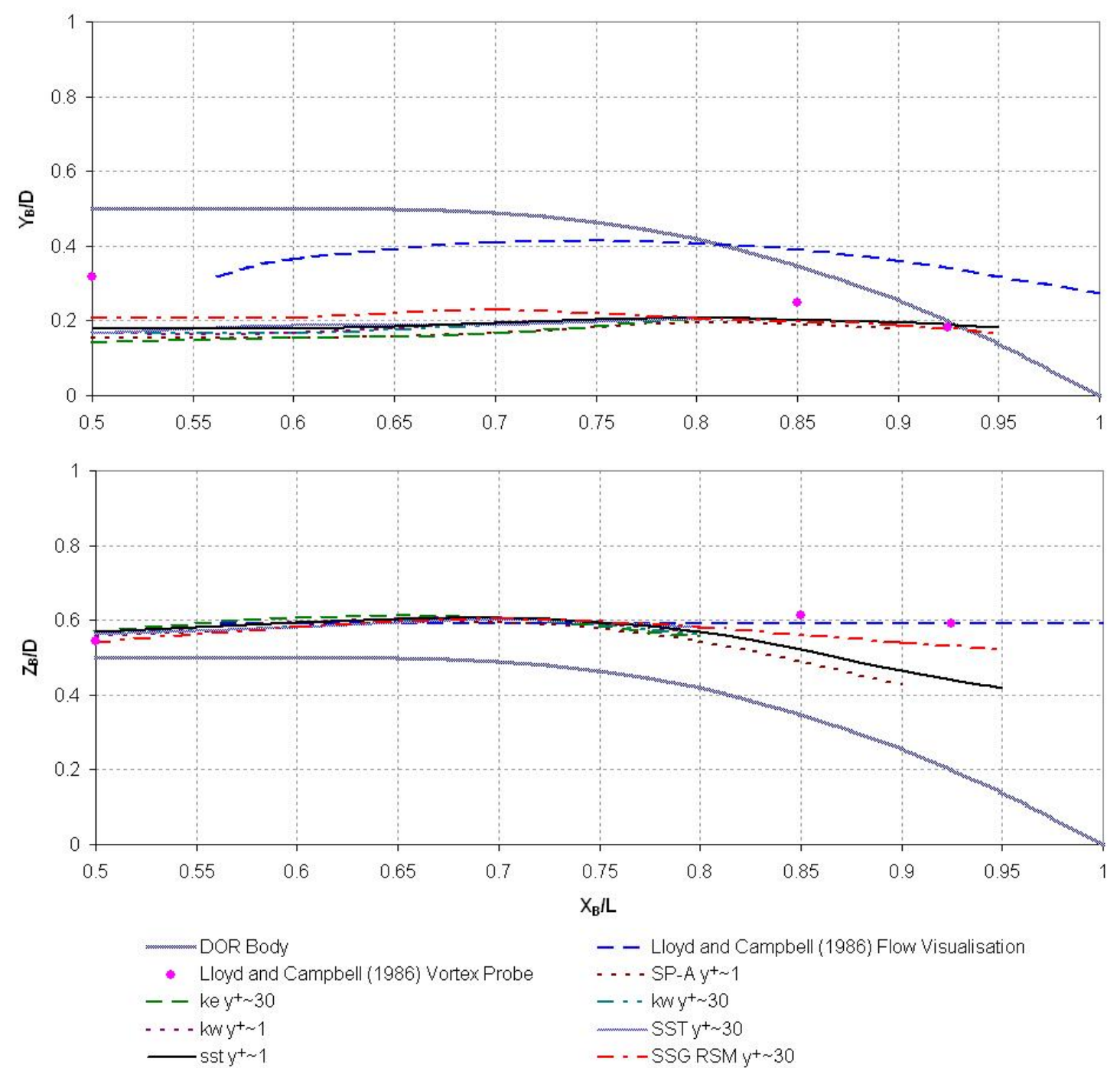

- - Lloyd and Campbell (1986) Flow Visualisation

-.. SP-A $\mathrm{y}^{+} 1$

- - $k W \mathrm{y}^{+} \sim 30$

SST $\mathrm{y}^{+} \sim 30$

- - SSG RSM y+ 30

Fig. 13 Comparison of estimated vortex centres from experimental and numerical results 


\subsubsection{Separation Line}

The 3D pressure field modifies the behaviour of the boundary layer around the hull leading to significant variation in the wall shear stress. Separation can be identified from the wall shear stress especially in regions of parallel mid-body where the pressure field remains constant. Over the mid-body for an attached flow the wall shear stress will drop with increasing boundary layer thickness. For a separating flow the wall stress will drop to a minimum at separation then increase as the separated region grows. The predicted longitudinal separation point, $X_{B 1}$, is relatively constant for all TCMs at $0.2 \mathrm{~L}$, see Figure 14.

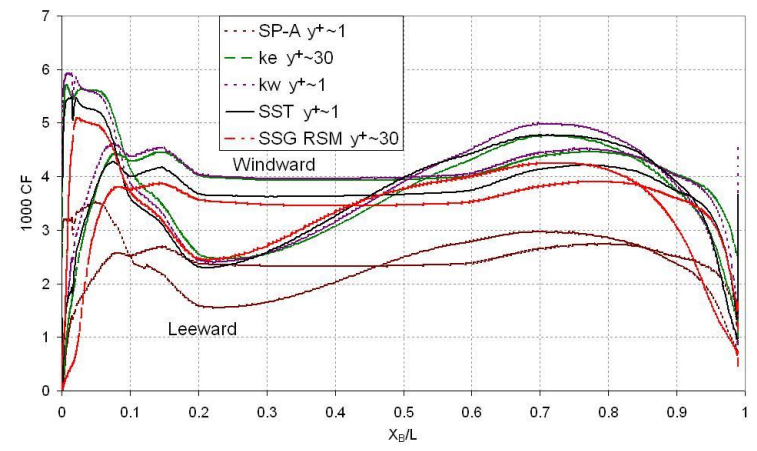

Fig. 14 Longitudinal skin friction distribution, $C F=$ $\frac{\sqrt{\tau_{x}^{2}+\tau_{y}^{2}+\tau_{z}^{2}}}{1 / 2 \rho U^{2}}$. For clarity of the figure, results for TCMs where simulations have been run for both with and without wall functions, only the without wall function case has been presented. Since, the variation is small compared to the differences between TCMs.

Wetzel et al. [28] compares experimental methods for identifying the crossflow separation line. Many of these approaches are relevant for interrogation of RANS data sets. To ease identification it is proposed to just consider the skin frictional coefficient in the crossflow direction in body fitted co-ordinates:

$C F_{\theta}=\frac{\left.\sqrt{(} \tau_{y}^{2}+\tau_{z}^{2}\right)}{1 / 2 \rho U^{2}}$

Results are presented in Figure 15. The separation point is identifiable as a local minima in the crossflow wall stress. Where the separation line runs roughly parallel to the body the minimum tends to zero. At the aft of the body were the separation line no longer runs roughly parallel to the body, the local crossflow velocity is no longer zero and hence there is a clear minimum but $C F_{\theta}$ does not drop to zero.

Figure 16 compares the separation line determined from the flow fields for the five TCMs. All five turbulence models show the separation occurring further to windward as the flow travels down the hull. The SSG RSM predicts separation furthest to windward, followed closely by the SST, while at approximately the same location the $k-\omega, k-\varepsilon$ and SP-A models predict separation up to $15^{\circ}$ further to leeward.

The extent of the separated region will have a direct influence on the pressure loading experienced by

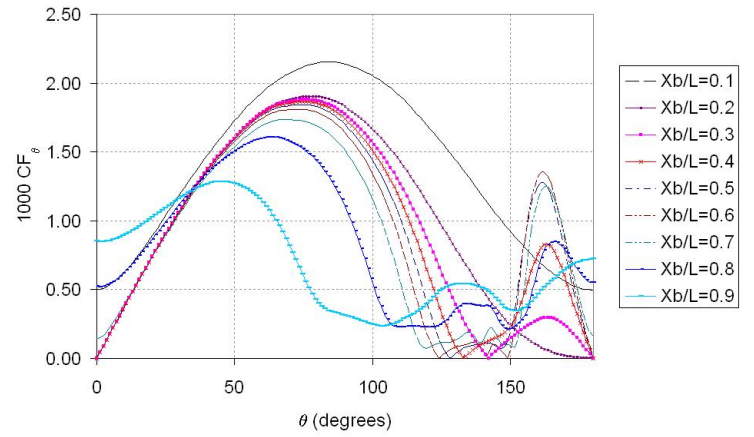

Fig. 15 SSG RSM $y^{+} 30 C F_{\theta}$ distribution at stations along the body. For clarity of the figure, results for TCMs where simulations have been run for both with and without wall functions, only the without wall function case has been presented. Since, the variation is small compared to the differences between TCMs.

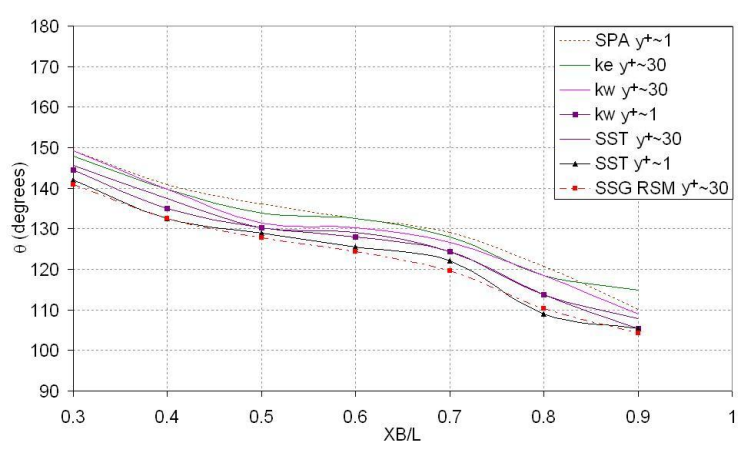

Fig. 16 Crossflow separation line for selected turbulence models.

the hull. This is reflected in the calculated pressure drag. The SSG RSM with the largest separated zone predicts the highest pressure drag of $50.91 \mathrm{~N}$ while the least separated case, SP-A, has a pressure drag of only $30.55 \mathrm{~N}$ with the remaining TCMs ranging between. Similarly, a larger separated lower pressure region in the aft leeward side of the hull will reduce the pitching moment acting on the body. The SSG RSM has the lowest pitching moment at $696.4 \mathrm{Nm}$ while the SP-A model has the highest at $765.80 \mathrm{Nm}$ with the remaining turbulence models appropriately arranged in order of separation line location.

The use of wall functions delays separation for roughly $5^{\circ}$ for both the SST and $k-\omega$ models compared with full resolution of the viscous sublayer. This reduction in separated zone leads to a reduction in pressure drag of approximately $5 \%$, a reduction in lift of approximately $5 \%$ and a increase in pitching moment of $1.5 \%$.

\section{Discussion}

Significant variations appear between the various turbulence closure models. In order to understand the differences it is useful to understand the dominant process acting on the fluid. This can be achieved by visualising the normalised invariant of the deformation tensor, $\mathcal{D}$ : 
$\mathcal{D}=\frac{S_{i j} S_{i j}-\Omega_{i j} \Omega_{i j}}{S_{i j} S_{i j}+\Omega_{i j} \Omega_{i j}}$

where $S_{i j}$ and $\Omega_{i j}$ are strain and rotation tensors defined by $S_{i j}=\left(\partial U_{i} / \partial x_{j}+\partial U_{j} / \partial x_{i}\right) / 2$ and $\Omega_{i j}=$ $\left(\partial U_{i} / \partial x_{j}-\partial U_{j} / \partial x_{i}\right) / 2$. $\mathcal{D}$ varies from -1 to 1 , where -1 indicates pure rotation, 0 indicates shear and 1 indicates strain. In a similar result to that presented by [9] for a prolate spheroid, Figure 17 illustrates contours of $\mathcal{D}$ at $X_{B} / L=0.925$. The flow is shear dominated $(\mathcal{D}=0)$ in the leeward boundary layer and surrounding the vortex core. In the centre of the primary vortex and in the core of the small counter rotating secondary vortex to leeward of the vortex sheet the flow is rotation dominated.

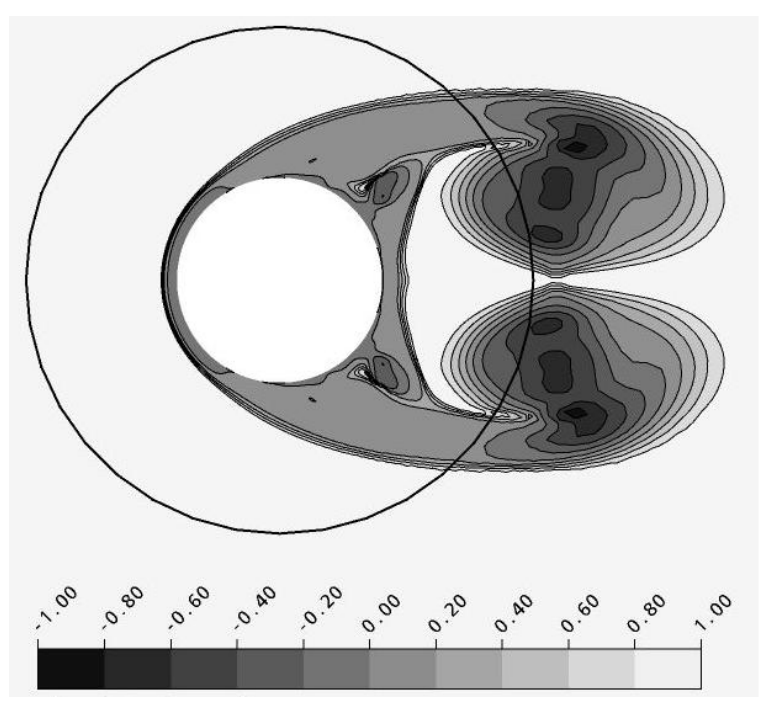

Fig. 17 Contours of the normalised invariant of the deformation tensor at $X_{B} / L=0.925$, results from SSG-RSM on VFine mesh.

Clearly the mean flow is highly $3 \mathrm{D}$ and the fluid experiences high levels of both strain and rotation which prove challenging for the TCMs. Also the eddy viscosity approach based on the Boussinesq approximation assumes that the turbulence shear stress angle is identical to the flow gradient angle. Chesnakas and Simpson [29] studied this assumption in the boundary layer of a prolate spheroid at $X / L=0.6$ at a Reynolds number of $4.2 \times 10^{6}$. At 10 degrees incidence they observed that the flow gradient angle and the shear stress angle are closely aligned. At 20 degrees incidence the shear stress angle and flow gradient angle are mainly aligned. However, under the primary vortex and in the vortex sheet the misalignment reaches greater than 90 degrees, indicating that in these regions the turbulence is anisotropic.

This suggests that the use of an eddy viscosity assumption may lead to erroneous results for a submarine hull at an incidence of $15^{\circ}$. This is reflected in the numerical results where the SSG-RSM appears to perform better than the eddy viscosity models in terms of predicting the path and strength of shed vortices.
Within the RANS formulation the influence of turbulence on the mean flow is represented by the Reynolds stress tensor $\rho \overline{u_{i}^{\prime} u_{j}^{\prime}}$. One equation turbulence models such as the Spalart Allmaras model only correctly model the principal Reynolds shear stresses, the normal Reynolds stresses are neglected. Two equation TCMs model both the normal and principle shear Reynolds stresses using the Boussinesq assumption (equation 3). For the SSG Reynolds Stress Model the Reynolds stresses are derived directly from six transport equations. Figure 18 compares the calculated normal Reynolds stresses at a transverse plane located at $X_{B} / L=0.925$, for the SST turbulence model (results are representative for all eddy viscosity models considered) and the SSG RSM. Significant Reynolds stresses are observed in the vortex sheet by all TCMs as it separates from the hull. However, the normal Reynolds stresses predicted by the two equation models differ significantly to the SSG RSM in the region of the vortex core, where the two equation models result in high Reynolds stresses and the SSG RSM predicts very small Reynolds stresses. Similarly looking at Figure 19 the vorticity $\zeta_{x}=\frac{\partial w}{\partial y}-\frac{\partial v}{\partial z}$ within the body vortex core is leading to modelled $\rho \overline{v^{\prime} w^{\prime}}$ in the two equation models which is not present in the results from the SSG-RSM. Clearly the presence of modelled Reynolds stresses in the core of the vortex for the one and two equation models will reduce the strength of the vortex, leading to a less-energetic flow characterised by a weak primary vortex.

\section{Conclusions}

Body vortices result in a non-linear relationship between lift and incidence angle due to the low pressure associated with the vortex core and its influence on the surface pressure. Therefore correct resolution of the forces and moments acting on a manoeuvring submarine requires accurate prediction of crossflow separation and of the path and strength of the body vortices.

The flow around a generic body of revolution submarine hull form has been calculated using RANS simulations with five turbulence closure models. Comparison between numerical and experimental results of the vortex structure and path have been presented. While the generic flow field predicted by all five models is similar local features vary leading to significant variation in the predicted global loads.

Vorticity traverses demonstrate that the SSG Reynolds stress model potentially provides an improvement in the prediction of both the vortex strength and path over conventional one and two equation turbulence closure models based on the eddy viscosity assumption. The computational cost of the SSG RSM is of the order of $250-300 \%$ of the conventional eddy viscosity approaches.

The eddy viscosity based methods under predict the vorticity within the body vortex by approximately $50 \%$. This is attributed to the Boussinesq approxi- 


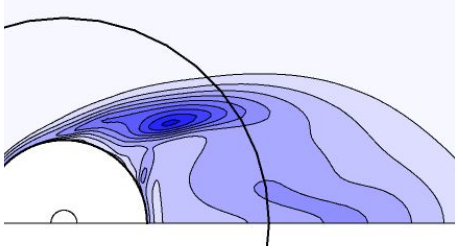

18(a): $\operatorname{SST} \rho \overline{u^{\prime} u^{\prime}}$

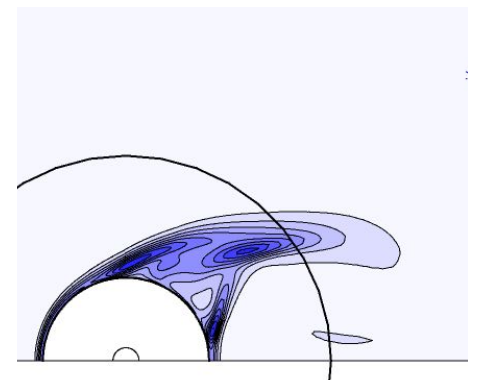

18(d): SSG RSM $\rho \overline{u^{\prime} u^{\prime}}$

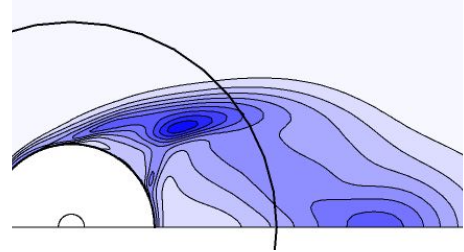

18(b): $\operatorname{SST} \rho \overline{v^{\prime} v^{\prime}}$

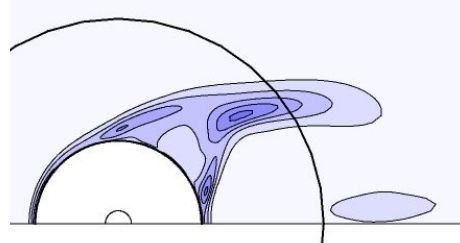

18(e): SSG RSM $\rho \overline{v^{\prime} v^{\prime}}$

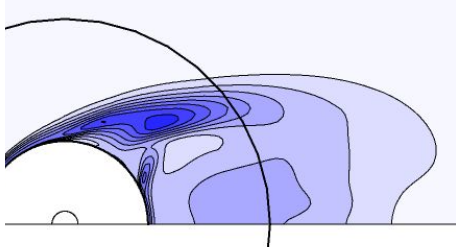

18(c): $\operatorname{SST} \rho \overline{w^{\prime} w^{\prime}}$

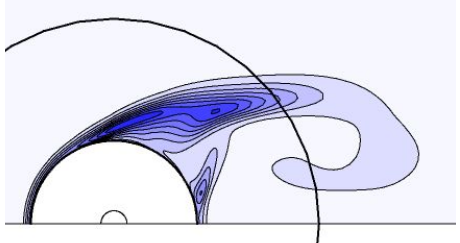

18(f): SSG RSM $\rho \overline{w^{\prime} w^{\prime}}$

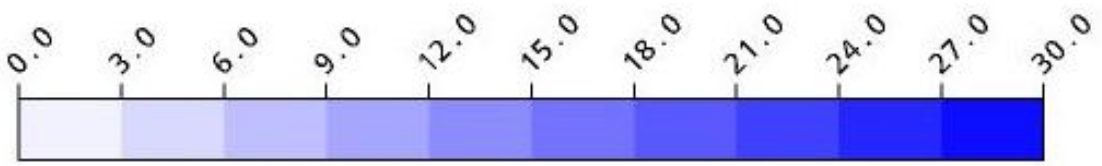

[Pa]

Fig. 18 Normal Reynolds stress contours, $X_{B} / L=0.925$.

mation which leads to significant Reynolds stresses within the vortex core not observed in the SSG RSM results leading to a less energetic flow. Consequently the use of Reynolds stress turbulence models is recommended to correctly capture the shed vortices developed around a manoeuvring underwater vehicle at intermediate incidence angles where symmetric crossflow body vortices are observed.

Acknowledgements The authors gratefully acknowledge the support of the School of Engineering Sciences and the National Oceanography Centre, Southampton in funding the $\mathrm{PhD}$ research of Mr A.B. Phillips as part of the on-going development programme of future AUVs for oceanographic exploration.

\section{References}

1. L.E. Ericsson and J.P. Reding. Tactical Missile Aerodynamics, chapter Asymmetric Vortex shedding from bodies of revolution. American Institute of Aeronautics and Astronautics, 1986.

2. A.R. Lloyd and I.M. Campbell. Experiments to investigate the vortices shed from a submarine like body. In 59th meeting of the AGARD fluid Dynamics Panel Symposium, 1986.

3. S. Lee, E. Jin, and H. Lee. Evaluation of the vertical plane stability by CFD. In Proceedings of the fifteenth(2005) International Polar Engineering Conference, Seoul Korea, June 19-24 2005.
4. B. Wu, F. Xing, X. Kuang, and O Mia. Investigation of hydrodynamic characteristics of submarine moving close to the sea bottom with CFD methods. Journal of Ship Mechanics, 9, 2005.

5. D. Bellevre, A. Diaz de Tuesta, and P. Perdon. Submarine manoeuvrability assessment using computational fluid dynamic tools. In Proc. 23th Symposium on Naval Hydrodynamics, Val de Reuil, France, pages 820-832, September 2000.

6. D. Boger and J. Dreyer. Prediction of hydrodynamic forces and moments for underwater vehicles using overset grids. In Proceedings of AIAA Aerospace Meeting and Exhibit Reno, Nevada, Jan 9-12, 2006.

7. K. Gee, R.M. Cummings, and L. Schiff. Turbulence model effects on separated flow about a prolate spheroid. AIAA Journal, 30:655-663, 1992.

8. G.S. Contantinescu, H. Pasinato, Y-Q Wang, J.R. Forsythe, and K.D. Squires. Numerical investigation of flow past a prolate spheroid. Transactions of ASME, 124:94-910, 2002.

9. S. Kim, Lebanon, N.H., S. Rhee, and D. Cokljat. Application of modern turbulence models to vortical flow around a prolate spheroid. In 41st Aerospace Sciences Meeting and Exhibit, Reno, Nevada, Jan. 6-9, 2003.

10. D.B. Clarke, P.A. Brandner, and G.J. Walker. Experimental and computational investigation of the flow around a 3-1 prolate spheroid. WSEAS Transactions of fluid mechanics, 3:207-217, 2008. 


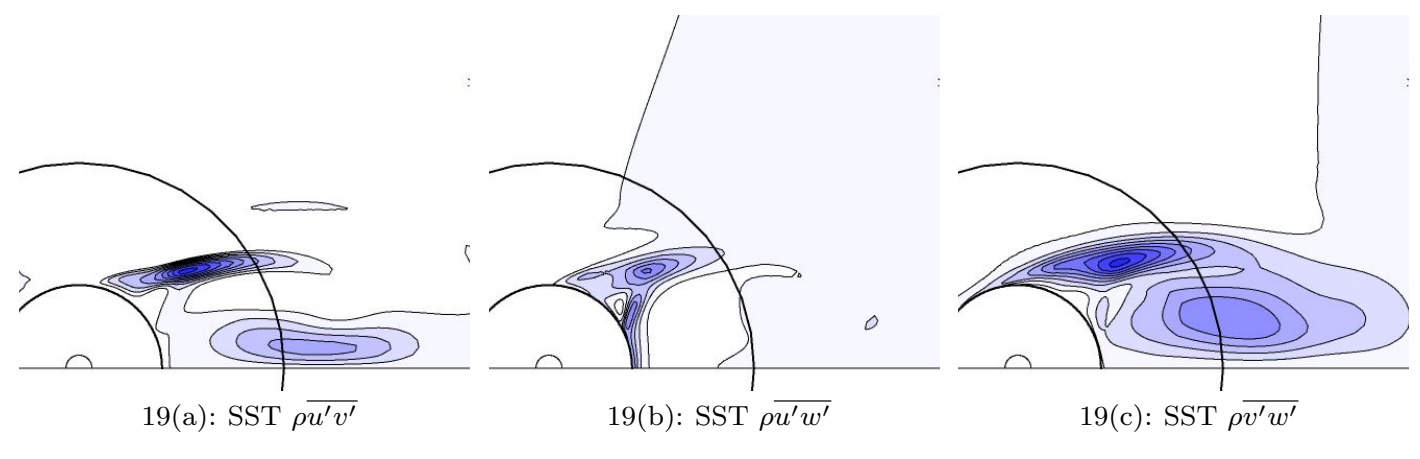

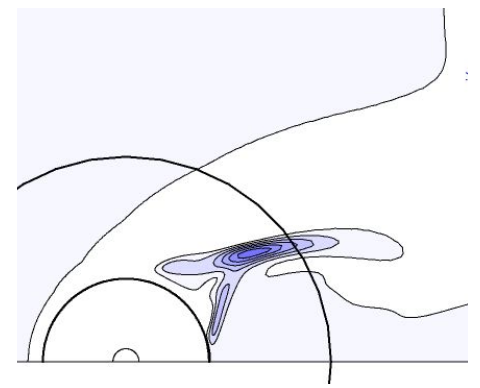

19(d): SSG RSM $\rho \overline{u^{\prime} v^{\prime}}$

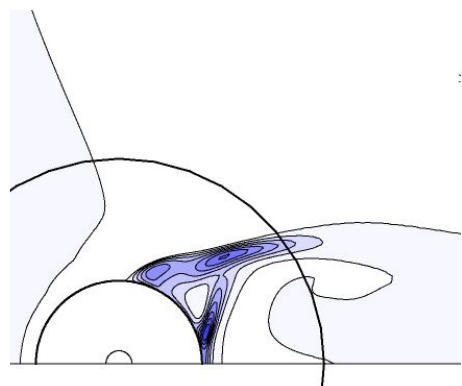

19(e): SSG RSM $\rho \overline{u^{\prime} w^{\prime}}$

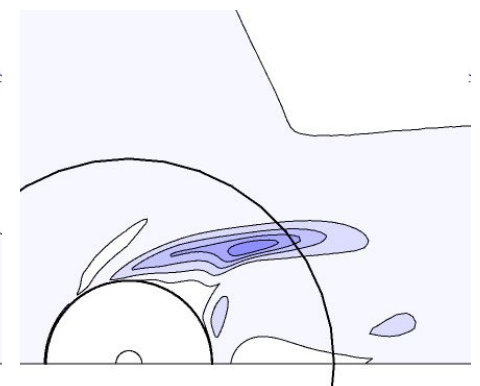

19(f): SSG RSM $\rho \overline{v^{\prime} w^{\prime}}$

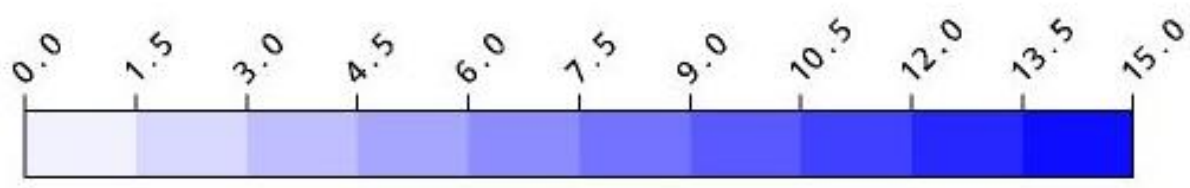

[Pa]

Fig. 19 Principle Reynolds Shear Stress Contours, $X_{B} / L=0.925$.

11. P.R. Spalart and S.R. Allmaras. A one-equation turbulence model for aerodynamic flows. In Aerospace Sciences Meeting and Exhibit, 30th, Reno, NV, Jan 6-9, 1992.

12. J.E. Bardina, P.G. Huang, and T.J. Coakley. Turbulence modelling validation, testing and development. Technical report, NASA Ames Research Centre, April, 1997.

13. ANSYS. ANSYS CFX, Release 11.0. ANSYS, 2006.

14. J.R. Edwards and S. Chandra. Comparrison of eddy viscosity-transport turbulence models for three-dimensional, shock-seperated flowfields. AIAA Journal, 34:756-763, 1996.

15. B.E. Launder and D.B. Spalding. The numerical computation of turbulent flows. Computer Methods in Applied Mechanics and Engineering, 3:269-289, 1974.

16. D. C. Wilcox. Reassessment of the scaledetermining equation for advanced turbulence models. AIAA journal, 26, 1988.

17. F.R. Menter. Two-equation eddy-viscosity turbulence models for engineering applications. AIAA Journal, 32(8):1598 - 605, 1994.

18. L. Larsson, F. Stern, and V. Bertram. Benchmarking of computational fluid dynamics for ship flows: The Gothenburg 2000 workshop. Journal of Ship Research, 47:63-81(19), 1 March 2003.

19. T. Hino. CFD workshop Tokyo 2005. In The Proceedings of CFD Workshop Tokyo, 2005.
20. C.G. Speziale, S. Sarkar, and T.B. Gatski. Modelling the pressure-strain correlation of turbulence: an invariant dynamical systems approach. J. Fluid Mechanics, 277:245-272, 1991.

21. T.J. Barth and D.C Jesperson. Design and application of upwind schemes on unstructured meshes. AIAA Paper 89-0366, 1989.

22. C.M. Rhie and W.L. Chow. A numerical study of the turbulent flow past an isolated airfoil with trailing edge separation. AIAA Paper 82-0998, 1982.

23. M.M. Freestone. Vorticity measurement by a pressure probe. Aeronautical Journal, 92:29-35, 1988.

24. F. Stern, R.V. Wilson, H.W. Coleman, and E.G. Paterson. Verification and validation of CFD simulations. Technical Report IIHR Report No. 407, Iowa Institute of Hydraulic Research, September 1999.

25. L.F. Richardson. The deferred approach to the limit. Transactions of the Royal society, London Series A, 226:223-361, 1927.

26. 3rd workshop on CFD uncertainty analysis, lisbon. 2008 .

27. C. Pashias. Propeller Tip Vortex Simulation Using Adaptive Grid Refinement Based On Flow Feature Identification. $\mathrm{PhD}$ thesis, University of Southampton, 2005.

28. T.G. Wetzel, R.L. Simpson, and C.J. Chesnakas. Measurement of three-dimensional crossflow sep- 
aration. AIAA Journal, 36:557-564, 1998.

29. C.J. Chesnakas and R. L. Simpson. Detailed investigation of the three-dimensional separation about a 6:1 prolate spheroid. AIAA Journal, 35:990-999, 1997. 